\title{
The Internal Symmetry Group of a Connection on a Principal Fiber Bundle with Applications to Gauge Field Theories
}

\author{
Arthur E. Fischer \\ Department of Mathematics, University of California, Santa Cruz, CA 95064, USA
}

\begin{abstract}
The internal symmetry group of a connection on a principal fiber bundle $P$ is studied. It is shown that this group is a smooth proper Lie transformation group of $P$, which, if $P$ is connected, is also free. Moreover, this group is shown to be isomorphic to the centralizer of the holonomy group of the connection. Several examples and applications of these results to gauge field theories are given.
\end{abstract}

\section{Contents}

Introduction . . . . . . . . . . . . . . . . . . . . 231

1. Notation and Preliminaries. . . . . . . . . . . . . . . . . . . . . 233

2. The Lie Group $I_{\omega}(P)$. . . . . . . . . . . . . . . . . . . . . . . . . . 240

3. Some Examples . . . . . . . . . . . . . . . . . . . . . . . . . . 244

4. Connections with Internal Symmetry Group as Large as Possible. . . . . . . . 250

5. Connections with Internal Symmetry Group as Small as Possible and Irreducible Connections . . . . . . . . . . . . . . . . . . . . . 252

6. $I_{\omega}(P)$ as a Lie Group of Transformations . . . . . . . . . . . . . . . . 256

7. The Lie Group of Transformations $I_{(q, \omega)}(P)$. . . . . . . . . . . . . . . . . . . . 259

References. . . . . . . . . . . . . . . . . . . . . 262

\section{Introduction}

Let $P(M, G)$ denote a principal fiber bundle with structure group $G$ over a connected manifold $M$, let AUT $(P)$ denote the group of automorphisms of $P$, and let $\operatorname{Aut}(P)$ denote the normal subgroup of automorphisms of $P$ that cover the identity diffeomorphism of $M$. Let $\omega$ be the connection 1 -form of a connection on $P$. If $F \in \operatorname{AUT}(P)$, let $F^{*} \omega$ denote the pullback of $\omega$ by $F$. Then

$$
\operatorname{AUT}_{\omega}(P)=\left\{F \in \operatorname{AUT}(P) \mid F^{*} \omega=\omega\right\}
$$

is the symmetry group of $\omega$, and

$$
l_{\omega}(P)=\left\{F \in \operatorname{Aut}(P) \mid F^{*} \omega=\omega\right\}=\operatorname{Aut}(P) \cap \operatorname{AUT}_{\omega}(P)
$$


is the internal symmetry group of $\omega$. In this paper we prove that if $P$ is a connected manifold, then with respect to the compact open topology, $I_{\omega}(P)$ is a finite dimensional Lie transformation group of $P$ that acts smoothly, properly, and freely on $P$ (see Theorem 6.2). This is in contrast to the fact that $\operatorname{AUT}_{\omega}(P)$ is in general an infinite-dimensional group. Another result is that for each $p \in P$, there is a Lie group isomorphism

$$
i_{p}: I_{\omega}(P) \rightarrow C_{G}\left(\operatorname{Hol}_{p}(\omega)\right), \quad F \mapsto u_{F}(p),
$$

where $\operatorname{Hol}_{p}(\omega) \subseteq G$ is the holonomy group of $\omega$ with reference point $p, C_{G}\left(\operatorname{Hol}_{p}(\omega)\right)$ is the centralizer of $\operatorname{Hol}_{p}(\omega)$ in $G$, and $u_{F}: P \rightarrow G$ is a map determined by $F$. Thus the determination of $I_{\omega}(P)$ is completely reduced to finding the holonomy group $\operatorname{Hol}(\omega)$ and an algebraic problem. We give several examples and applications of these results to gauge field theories.

It is of interest to compare these results to the results regarding the classical affine group of a linear connection $\Gamma$ on the frame bundle $L M$ of $M$. Let $\omega$ be the corresponding connection 1-form. Then a diffeomorphism $f$ of $M$ is an affine transformation if the induced mapping $T f: T M \rightarrow T M$ maps horizontal curves to horizontal curves. This condition is equivalent to $\hat{f}^{*} \omega=\omega$, where $\hat{f}: L M \rightarrow L M$ denotes the natural lift of $f: M \rightarrow M$ to the frame bundle $L M$. Thus if $\operatorname{Diff}(M)$ denotes the group of diffeomorphisms of $M$, then

$$
A_{\omega}(M)=\left\{f \in \operatorname{Diff}(M) \mid \hat{f}^{*} \omega=\omega\right\}
$$

is the group of affine transformations of $\omega$.

Now let $\theta$ denote the canonical $\mathbb{R}^{n}$-valued 1 -form on $L M$, and let

$$
\operatorname{AUT}_{\theta}(L M)=\left\{F \in \operatorname{AUT}(L M) \mid F^{*} \theta=\theta\right\} .
$$

Then $F \in \operatorname{AUT}_{\theta}(L M)$ if and only if $F=\widehat{f}$ for some $f \in \operatorname{Diff}(M)$ (Kobayashi-Nomizu [1963], p. 226, or Kobayashi [1972], p. 40). Thus

$$
\operatorname{AUT}_{\theta}(L M)=\left\{\hat{f} \in \operatorname{AUT}_{\theta}(L M) \mid f \in \operatorname{Diff}(M)\right\} \approx \operatorname{Diff}(M)
$$

and the affine group $A_{\omega}(M)$ is isomorphic to

$$
\begin{aligned}
A_{\omega}(M) \approx \operatorname{AUT}_{(\theta, \omega)}(L M) & =\left\{F \in \operatorname{AUT}(L M) \mid F^{*} \theta=\theta \text { and } F^{*} \omega=\omega\right\} \\
& =\operatorname{AUT}_{\theta}(L M) \cap \operatorname{AUT}_{\omega}(L M) .
\end{aligned}
$$

Thus at the bundle level, affine transformations are bundle automorphisms that preserve both the connection form $\omega$ and the canonical form $\theta$, so that the classical group of affine transformations is actually the simultaneous symmetry group of both $\omega$ and $\theta$. Although in general both $\operatorname{AUT}_{\theta}(L M)$ and $\operatorname{AUT}_{\omega}(L M)$ are infinite dimensional, the intersection $\operatorname{AUT}_{(\theta, \omega)}(L M) \approx A_{\omega}(M)$ is a finite dimensional Lie group.

It is interesting that historically affine transformations were first considered as transformations of $M$ whose natural lifts preserved $\omega$, rather than as more general bundle automorphisms of $L M$ that preserve $\omega$, thereby relegating the interesting infinite dimensional group $\operatorname{AUT}_{\omega}(L M)$ to the shelf of mathematical obscurities.

On a general principal fiber bundle there is no canonical 1-form $\theta$. However, there are still interesting "reductions" of $\operatorname{AUT}_{\omega}(P)$ resulting in a finite dimensional 
group. Firstly, we may intersect $\operatorname{AUT}_{\omega}(P)$ with $\operatorname{Aut}(P)$, thereby obtaining the internal symmetry group $I_{\omega}(P)$. Thus in this respect $\operatorname{Aut}(P)$ plays the role $\operatorname{AUT}_{\theta}(L M)$ did for the affine connection on the frame bundle $L M$.

Another possibility is to put a Riemannian metric $g$ on $M$ and consider the group

$$
I_{(g, \omega)}(P)=\left\{F \in \operatorname{AUT}(P) \mid F^{*} \omega=\omega \text { and } f^{*} g=g\right\},
$$

where $f$ is the diffeomorphism of $M$ induced by the automorphism $F$. Thus $I_{(g, \omega)}(P)$ is the group of symmetries of $\omega$ that cover isometries of $g$. This also is a finite dimensional Lie transformation group of $P$ acting smoothly, properly, and effectively (though not freely) on $P$ (see Theorem 7.1). This group is of interest in gauge field theories (see Fischer [1982, 1985, 1988] and the references therein).

On a frame bundle $L M$ we can compare the internal symmetry group

$$
I_{\omega}(L M)=\operatorname{Aut}(L M) \cap \operatorname{AUT}_{\omega}(L M)
$$

with the affine transformation group

$$
A_{\omega}(M) \approx \operatorname{AUT}_{(\theta, \omega)}(L M)=\operatorname{Aut}_{\theta}(L M) \cap \operatorname{AUT}_{\omega}(L M) .
$$

But if $F \in I_{\omega}(L M) \cap \operatorname{AUT}_{(\theta, \omega)}(L M), F$ must cover the identity diffeomorphism id ${ }_{M}$ of $M$ [since $\left.I_{\omega}(L M) \subseteq \operatorname{Aut}(L M)\right]$, and $F$ must be the lift of a diffeomorphism of $M$ [since $\left.\operatorname{AUT}_{(\theta, \omega)} \subseteq \mathrm{AUT}_{\theta}(L M)\right]$. Thus $F=\mathrm{id}_{L M}$, the identity diffeomorphism of $L M$, and so

$$
I_{\omega}(L M) \cap \operatorname{AUT}_{(\theta, \omega)}(L M)=\left\{\operatorname{id}_{L M}\right\} .
$$

Thus the internal symmetry group $I_{\omega}(L M)$ has only a trivial intersection with $\operatorname{AUT}_{(\theta, \omega)}(L M) \approx A_{\omega}(M)$. Thus $I_{\omega}(L M)$ and $\operatorname{AUT}_{(\theta, \omega)}(L, M)$ are respectively the "vertical" and "horizontal" components of $\operatorname{AUT}_{\omega}(L M) . I_{\omega}(L M)$ itself has no classical analog.

In considering the symmetry (or automorphism) group of a geometric structure (see Kobayashi-Nomizu [1963], p. 306, and [1969], p. 392), attention is usually focused on the automorphism group of a $G$-structure on $M$, i.e., a reduction of $L M$ to a $G$-principal fiber bundle, $G \subseteq G L(n)$. The automorphism group is then the subgroup of $\operatorname{Diff}(M)$ consisting of those maps $f$ whose natural lift $\hat{f}$ maps the $G$-structure to itself. In this regard the affine group $A_{\omega}(M)$ of a linear connection $\omega$ is somewhat of an exception inasmuch as a connection is not a $G$-structure on $M$ (i.e., it is not a reduction of $L M$ ), and so $A_{\omega}(M)$ is not an automorphism group of a $G$-structure. Similarly, a connection $\omega$ on a general principal fiber bundle $P(M, G)$ is a geometric structure on $P$ (or on $M$ ), but is not a $G$-structure on $M$, and so the internal symmetry group $I_{\omega}(P)$ is not an automorphism group of a $G$-structure. However, $I_{\omega}(P)$ is a symmetry group of a geometric structure, and so our result that $I_{\omega}(P)$ is a Lie transformation group adds another geometric structure to the list enumerated in Kobayashi-Nomizu [1969], p. 332, of geometric structures whose automorphism groups are Lie groups.

\section{Notation and Preliminaries}

Throughout this paper, $M$ will denote a connected $C^{\infty} n$-dimensional manifold (Hausdorff and second countable, and hence paracompact), $G$ a (second countable) 
Lie group, and $P(M, G)$ a principal fiber bundle (PFB) with total space $P$, base space $M$, structure group $G$, projection map

and right action

$$
\pi: P \rightarrow M,
$$

$$
\Phi: P \times G \rightarrow P, \quad(p, a) \mapsto p \cdot a .
$$

All of the above paraphernalia will be denoted by $P(M, G)$.

For $p \in P$, let

$$
\Phi_{p}: G \rightarrow P, \quad a \mapsto p \cdot a,
$$

denote the orbit map through $p$, and for $a \in G$, let

$$
R_{a}=\Phi_{a}: P \rightarrow P, \quad p \mapsto p \cdot a
$$

denote the diffeomorphism of $P$ corresponding to $a \in G$. Let $G(P)$ $=\left\{R_{a}: P \rightarrow P \mid a \in G\right\}$ denote the Lie group of diffeomorphisms of $P$ induced by the action $\Phi$.

Let $e$ be the identity in $G$, let $\left(\mathfrak{y}=T_{e} G\right.$ the Lie algebra of $G$ (taken with the usual Lic algebra structure of left-invariant vector fields on $G$ ), and let

$$
\text { Ad : } G \rightarrow G L((\mathfrak{5}), \quad a \mapsto \operatorname{Ad}(a):(\mathfrak{5} \rightarrow \mathbb{6}
$$

denote the adjoint representation of $G$ on $\left(\mathfrak{5}\right.$. Let $V_{p}=\operatorname{ker} T_{p} \pi \cong T_{p} P$ denote the vertical subspace of the tangent space $T_{p} P$, and let $T_{e} \Phi_{p}:\left(\mathfrak{5} \rightarrow V_{p} \subseteq T_{p} P\right.$ denote the derivative at the identity $e \in G$ of the orbit map $\Phi_{p}: G \rightarrow P$. For $A \in \mathbf{6}$, let $A^{*}$ be the fundamental vector field on $P$ generated by $A$. For $p \in P, A^{*}$ is defined by $A^{*}(p)$ $=T_{e} \Phi_{p} \cdot A$.

Let $\operatorname{Diff}(P)$ and $\operatorname{Diff}(M)$ denote the groups of $C^{\infty}$ diffeomorphisms of $P$ and $M$, respectively, with composition of diffeomorphisms as the group structure. Let

$$
\operatorname{AUT}(P)=\left\{F \in \operatorname{Diff}(P) \mid F \circ R_{a}=R_{a} \circ F \text { for all } a \in G\right\}
$$

denote the group of automorphisms of $P$. The condition $F \circ R_{a}=R_{a} \circ F$ can also be written $F(p \cdot a)=F(p) \cdot a$ for all $p \in P$ and $a \in G$. For $F \in \operatorname{AUT}(P), F$ maps fibers to fibers and so induces a diffeomorphism of the base manifold $M$. Let $f=\hat{\pi}(F) \in \operatorname{Diff}(M)$ denote this induced diffeomorphism. Morever, the map

$$
\hat{\pi}: \operatorname{AUT}(P) \rightarrow \operatorname{Diff}(M), \quad F \rightarrow \hat{\pi}(F)=f,
$$

is a group homomorphism. Its kernel is

$$
\operatorname{Aut}(P)=\left\{F \in \operatorname{AUT}(P) \mid \hat{\pi}(F)=\mathrm{id}_{M}\right\},
$$

the normal subgroup of automorphisms of $P$ covering the identity diffeomorphism $\mathrm{id}_{M}$ of $M$. The group $\operatorname{Aut}(P)$ has two other representations, which we briefly describe.

Firstly, let $C^{\infty}(P, G)$ denote the $C^{\infty}$ maps from $P$ to $G$, and let

$$
C_{\mathrm{Aut}}^{\infty}(P, G)=\left\{u \in C^{x}(P, G) \mid u(p \cdot a)=a^{-1} \cdot u(p) \cdot a \text { for all } p \in P \text { and } a \in G\right\} .
$$

Then $C_{\text {Aut }}^{\infty}(P, G)$ is a group with respect to pointwise multiplication of functions.

Secondly, consider the left action of $G$ on itself by inner automorphisms $G \times G \rightarrow G,(a, b) \rightarrow a \cdot b \cdot a^{-1}$. Let $G_{\text {Aut }}(M)=(P \times G) / G$ denote the corresponding 
associated fiber bundle over $M$ with standard fiber $G$. For $x \in M$, the fiber at $x$, denoted $G_{\text {Aut }}(M)_{x}$, is the Lie group of diffeomorphisms of the fiber $\pi^{-1}(x) \subseteq P$ that commute with the action of $G$ on $\pi^{-1}(x)$. Thus $G_{\text {Aut }}(M)$ is a bundle of Lie groups over $M$. Let $C^{\infty}\left(G_{\text {Aut }}(M)\right)$ denote the space of smooth cross-sections of $G_{\text {Aut }}(M)$, taken with its group structure of pointwise multiplication of sections. Then the following proposition is basic (see e.g., Trautman [1980], or Fischer [1985]).

1.1. Proposition. The three groups $\operatorname{Aut}(P), C_{\text {Aut }}^{\infty}(P ; G)$ and $C^{\infty}\left(G_{\text {Aut }}(M)\right)$ are naturally isomorphic with each other. The first of these isomorphisms (i.e., group bijections) is given by

$$
\operatorname{Aut}(P) \rightarrow C_{\mathrm{Aut}}^{\infty}(P, G), \quad F \mapsto u_{F},
$$

where for $p \in P, u_{F}(p)$ is defined by the equation

$$
F(p)=p \cdot u_{F}(p)
$$

The second of these isomorphisms is given by

$$
C_{\text {Aut }}^{\infty}(P, G) \rightarrow C^{\infty}\left(G_{\text {Aut }}(M)\right), \quad u \mapsto u_{M},
$$

where for $x \in M, u_{M}(x)$ is defined $b y$

$$
u_{M}(x)=\left\{\left(p \cdot a, a^{-1} \cdot u(p) \cdot a\right) \mid a \in G\right\} \in(P \times G) / G,
$$

where $p \in \pi^{-1}(x)$, and the above definition is independent of the choice of $p$.

We shall have occasion to use the viewpoint represented by all three groups. However, since we shall be working primarily on $P$, we shall use mostly either $\operatorname{Aut}(P)$ or $C_{\text {Aut }}^{\infty}(P, G)$, depending on convenience. In Sect. 2, convenience will dictate strongly that $C_{\mathrm{Aut}}^{\infty}(P, G)$ be primarily used, while in $\operatorname{Sect}$. 6, Aut $(P)$ will be primarily used.

Let $\mathfrak{X}(P)$ and $\mathfrak{X}(M)$ denote the Lie algebras of $C^{\infty}$ vector fields on $P$ and $M$, respectively, taken with the usual bracket of vector fields as the Lie algebra structure. Let

$$
\mathfrak{X}_{G}(P)=\left\{Z \in \mathfrak{X}(P) \mid\left(R_{a}\right)_{*} Z=Z \text { for all } a \in G\right\}
$$

denote the Lie subalgebra of $G$-invariant vector fields on $P$. Then $\pi: P \rightarrow M$ induces a Lie algebra homomorphism $\pi_{*}: \mathfrak{X}_{G}(P) \rightarrow \mathfrak{X}(M), Z \mapsto \pi_{*} Z=X$, whose kernel is given by

$$
\mathfrak{X}_{G}^{\mathrm{vert}}(P)=\left\{Z \in \mathfrak{X}_{G}(P) \mid \pi_{*} Z=0\right\},
$$

the $G$-invariant vertical vector fields on $P$. As above, $\mathfrak{X}_{G}^{\text {vert }}(P)$ has two other representations. Let $C^{x}\left(P,(5)\right.$ denote the $C^{\infty}$ maps from $P$ to $(5)$, and let

$$
C_{\mathrm{Ad}}^{\infty}\left(P,(\mathfrak{b})=\left\{\varphi \in C^{\infty}\left(P,(\mathfrak{b}) \mid \varphi(p \cdot a)=\operatorname{Ad}\left(a^{-1}\right) \cdot \varphi(p) \text { for all } P \in P \text { and } a \in G\right\} .\right.\right.
$$

Then $C_{\mathrm{Ad}}^{\infty}(P,(5)$ is a Lie algebra using the pointwise bracket of maps, i.e., $\left[\varphi_{1}, \varphi_{2}\right](p)=\left[\varphi_{1}(p), \varphi_{2}(p)\right]$. Also, consider the adjoint action of $G$ on $\mathbf{6}$, $G \times\left(\mathfrak{5} \rightarrow\left(\mathfrak{5},(a, A) \rightarrow \operatorname{Ad}(a) \cdot A\right.\right.$, and let $\mathfrak{6}_{\mathrm{Ad}}(M)=(P \times(\mathfrak{5}) / G$ denote the corresponding associated vector bundle over $M$ with standard fiber $\left(\mathfrak{5}\right.$. Then $\left(\mathfrak{5}_{\mathrm{Ad}}(M)\right.$ is a 
bundle of Lie algebras over $M$. Let $C^{\infty}\left(\mathfrak{5}_{\mathrm{Ad}}(M)\right)$ denote the space of $C^{\infty}$ crosssections. Then $C^{\infty}\left(\boldsymbol{5}_{\mathrm{Ad}}(M)\right)$ is a Lie algebra with respect to the pointwise bracket of sections. Corresponding to Proposition 1.1 is the following.

1.2. Proposition. The three Lie algebras $\mathfrak{X}_{G}^{v e r t}(P), C_{\mathrm{Ad}}^{\infty}\left(P,(\mathfrak{5})\right.$, and $C^{\infty}\left(\mathfrak{5}_{\mathrm{Ad}}(M)\right)$ are naturally isomorphic with each other. These Lie algebra isomorphisms (i.e., Lie algebra bijections) are given respectively, by

$$
\mathfrak{X}_{G}^{\text {vert }}(P) \rightarrow C_{\mathrm{Ad}}^{\infty}\left(P ;(\mathfrak{5}), \quad T \mapsto \varphi_{T},\right.
$$

where for $p \in P, \varphi_{T}(p)$ is defined by the equation $T_{e} \Phi_{p} \cdot \varphi_{T}(p)=T(p)$, and by

$$
C_{\mathrm{Ad}}^{\infty}\left(P,(\mathfrak{5}) \rightarrow C^{\infty}\left(\mathfrak{5}_{\mathrm{Ad}}(M)\right), \quad \varphi \mapsto \varphi_{M},\right.
$$

where for $x \in M, \varphi_{M}(x)$ is defined by

$$
\varphi_{M}(x)=\left\{\left(p \cdot a, \operatorname{Ad}\left(a^{-1}\right) \cdot \varphi(p)\right) \mid a \in G\right\} \in(P \times(\mathfrak{b}) / G,
$$

where $p \in \pi^{-1}(x)$, and which is independent of the choice of $p$.

Let $\exp :(5) G$ denote the exponential map of the Lie algebra $(5$. Then exp induces an exponential map

$$
\mathrm{EXP}: C_{\mathrm{Ad}}^{\infty}\left(P,(\mathfrak{5}) \rightarrow C_{\mathrm{Aut}}^{\infty}(P, G),\right.
$$

where if $\varphi \in C_{\mathrm{Ad}}^{\infty}(P ;(\mathfrak{5}), \mathrm{EXP}$ is defined pointwise by $(\operatorname{EXP} \varphi)(p)=\exp (\varphi(p))$. The resulting $u=\operatorname{EXP} \varphi$ is in $C_{\text {Aut }}^{\infty}(P, G)$, since

We let

$$
\begin{aligned}
u(p \cdot a) & =(\operatorname{EXP} \varphi)(p \cdot a)=\exp (\varphi(p \cdot a))=\exp \left(\operatorname{Ad}\left(a^{-1}\right) \cdot \varphi(p)\right) \\
& =a^{-1} \cdot \exp (\varphi(p)) \cdot a=a^{-1} \cdot u(p) \cdot a .
\end{aligned}
$$

$$
\operatorname{Exp}: \mathfrak{X}_{G}^{\mathrm{vert}}(P) \rightarrow \operatorname{Aut}(P), \quad T \mapsto \operatorname{Exp} T
$$

denote the corresponding exponential map on $\mathfrak{X}_{G}^{\text {vert }}(P)$, defined by

$$
(\operatorname{Exp} T)(p)=p \cdot\left(\operatorname{EXP} \varphi_{T}\right)(p)=p \cdot\left(\exp \varphi_{T}(p)\right) .
$$

Note that if $\varphi \in C_{\mathrm{Ad}}^{\infty}\left(P,(\mathfrak{G})\right.$, or $\left.T \in \mathfrak{X}_{G}^{\mathrm{vert}}(P)\right)$, then $u_{\lambda}=\operatorname{EXP}(\lambda \varphi) \in C_{\text {Aut }}^{\infty}(P, G)$ and $F_{\lambda}=\operatorname{Exp} \lambda T \in \operatorname{Aut}(P)$ are defined for all $\lambda \in \mathbb{R}$. Thus, in particular, if $T \in \mathfrak{X}_{G}^{\text {vert }}(P), T$ is a complete vector field on $P$ with flow $F_{\lambda}=\operatorname{Exp} \lambda T$.

It is important to note that, in general, the diffeomorphism $R_{a}: P \rightarrow P, a \in G$, is not an automorphism of $P$. Indeed, $R_{a} \in \operatorname{Aut}(P)$ if and only if $a$ is in the center $Z(G)$ of $G$, where the center

$$
Z(G)=\{a \in G \mid a \cdot b=b \cdot a \text { for all } b \in G\}
$$

is a closed normal subgroup of $G$, and hence a Lie subgroup. Note that $Z(G) \cong$ ker Ad, and if $G$ is connected, then we have equality.

The Lie algebra of $Z(G)$ is

$$
z(G)=\{A \in \mathfrak{G} \mid \operatorname{Ad}(b) \cdot A=A \text { for all } b \in G\} .
$$

On the other hand, the center of the Lie algebra 6 is defined to be

$$
z(\mathfrak{G})=\{A \in \mathfrak{G} \mid[A, B]=0 \text { for all } B \in \mathfrak{G}\},
$$

so that $z(G) \subseteq z((\mathfrak{5})$. If $G$ is connected, then $z(G)=z((\mathfrak{5})$. 
Let

$$
Z_{G}(P)=\left\{R_{a} \mid a \in Z(G)\right\}=G(P) \cap \operatorname{Aut}(P),
$$

a normal subgroup of $\operatorname{Aut}(P)$. Similarly, let $Z_{G}(P)=\left\{A^{*} \in \mathfrak{X}_{G}^{\text {vert }}(P) \mid A \in z(G)\right\}$, where $A^{*}$ denotes the fundamental vector field on $P$ associated with $A \in(\mathfrak{5}$, denote the corresponding ideal of $\mathfrak{X}_{G}^{\mathrm{vert}}(P)$. Note that if $A \in z(G)$, then $\operatorname{Ad}(a) \cdot A=A$ for all $a \in G$, and so

$$
\left(R_{a}\right)_{*} A^{*}=\left(\operatorname{Ad}\left(a^{-1}\right) \cdot A\right)^{*}=A^{*},
$$

so that $A^{*}$ is indeed in $\mathfrak{X}_{G}^{\text {vert }}(P)$. For $A \notin Z(G), A^{*} \in \mathfrak{X}^{\text {vert }}(P)$, but is not $G$-invariant.

Similarly, let

$$
Z(P, G)=\{u: P \rightarrow Z(G) \mid u \text { is constant on } P\},
$$

and

$$
Z(P, \mathfrak{5})=\{\varphi: P \rightarrow z(G) \mid \varphi \text { is constant on } P\},
$$

the corresponding normal subgroup and ideal of $C_{\mathrm{Aut}}^{\infty}(P, G)$ and $C_{\mathrm{Add}}^{\infty}(P,(\mathfrak{5})$, respectively. Note that if $u \equiv$ constant $\in Z(G)$, then $u(p \cdot a)=a^{-1} \cdot u(p) \cdot a=u(p)$, so $u$ is in $C_{\mathrm{Aut}}^{\infty}(P, G)$, and similarly, if $\varphi \equiv$ constant $\in z(G)$, then

$$
\varphi(\mathrm{p} \cdot \mathrm{a})=\operatorname{Ad}\left(a^{-1}\right) \cdot \varphi(p)=\varphi(p),
$$

So $\varphi$ is in $C_{\mathrm{Ad}}^{\infty}(P,(5)$.

Let

$$
Z\left(C_{\text {Aut }}^{\infty}(P, G)\right)=\left\{u \in C_{\text {Aut }}^{\infty}(P, G) \mid u \cdot u_{1}=u_{1} \cdot u \text { for all } u_{1} \in C_{\text {Aut }}^{\infty}(P, G)\right\}
$$

denote the center of $C_{\mathrm{Aut}}^{\infty}(P, G)$. Since for fixed $p$ and varying $u_{1}, u_{1}(p)$ spans $G$, $u \in Z\left(C_{\mathrm{Aut}}^{\infty}(P, G)\right)$ if and only if $u$ takes values only in $Z(G)$ if and only if $u$ is constant on the fibers of $P$. Such a $u$ may however vary from fiber to fiber. Thus

$$
Z\left(C_{\text {Aut }}^{\infty}(P, G)\right)=C_{\text {Aut }}^{\infty}(P, Z(G))=\left\{u \in C_{\text {Aut }}^{\infty}(P, G) \mid u(p) \in Z(G) \text { for all } p \in P\right\},
$$

which in general is an infinite dimensional subgroup of $C_{\text {Aut }}^{\infty}(P, G)$. If however $G$ is a semi-simple Lie group, then its center $Z(G)$ is a discrete subgroup of $G$. Thus if $\left.u \in C_{\text {Aut }}^{\infty}(G)\right), u$ is constant on fibers, and by continuity of $u$ and connectivity of $M, u$ is then constant on $P$. Thus in this case, the centers of $C_{\text {Aut }}^{\infty}(P, G)$ and $G$ are isomorphic

$$
Z\left(C_{\text {Aut }}^{\infty}(P, G)\right)=Z(P, G) \approx Z_{G}(P) \approx Z(G),
$$

and are zero dimensional Lie groups (see also Daniel-Viallet [1980]).

For $0 \leqq k \leqq n=\operatorname{dim} M$, let $C^{\infty}\left(\Lambda^{k}(P) \otimes(\mathfrak{5})\right.$ denote the space of smooth Liealgebra valued $k$-forms on $P$, let

$$
C_{\mathrm{Ad}}^{\infty}\left(\Lambda^{k}(P) \otimes(\mathfrak{5})=\left\{\varphi \in C^{\infty}\left(\Lambda^{k}(P) \otimes(\mathfrak{5}) \mid R_{a}^{*} \varphi=\operatorname{Ad}\left(a^{*}\right) \cdot \varphi \text { for all } a \in G\right\}\right.\right.
$$

denote the Lie algebra-valued $k$-forms of type (Ad, (5), and let

$$
\begin{aligned}
A^{k}(P,(\mathfrak{5}) & =C_{\mathrm{Ad} . \text { hor }}^{\infty}\left(\Lambda^{n}(P) \otimes(\mathfrak{5})\right. \\
& =\left\{\varphi \in \mathrm{C}_{\mathrm{Ad}, \text { hor }}^{\infty}\left(\Lambda^{k}(P) \otimes(\mathfrak{5}) \mid \varphi\left(Z_{1}, \ldots, Z_{k}\right)=0 \text { whenever one of } Z_{i} \text { 's is vertical }\right\}\right.
\end{aligned}
$$

denote the space of horizontal (or tensorial) Lie-algebra valued $k$-forms of type $\left(\mathrm{Ad},(\mathfrak{5})\right.$. Since a zero-form on $P$ is always horizontal, $C_{\mathrm{Ad}}^{\infty}(P, \mathfrak{5})=A^{0}(P,(\mathfrak{5})$. 
A connection 1-form $\omega$ on $P$ is an element of $C^{\infty}\left(\Lambda^{1}(P) \otimes(5)\right.$ such that

(1) $R_{a}^{*} \omega=\operatorname{Ad}\left(a^{-1}\right) \cdot \omega$ for all $a \in G$;

(2) $\omega\left(A^{*}\right)=A$ for all $A \in \mathbf{b}$.

We let $\mathscr{C}(P)$ denote the space of connection 1 -forms on $P$, naturally identifiable with the space of connections on $P$. Since $M$ is paracompact, $\mathscr{C}(P) \neq \emptyset$ (see Kobayashi-Nomizu [1967], p. 67). Also, $\mathscr{C}(P)$ is an affine space, and is the affine space associated to the vector space $A^{1}(P,(\mathfrak{5})$.

For a connection 1-form $\omega$, we let

$$
D: C_{\mathrm{Ad}}^{\infty}\left(P,(\mathfrak{5}) \rightarrow A^{1}(P,(\mathfrak{5}), \quad \varphi \mapsto D \varphi\right.
$$

denote the gauge covariant exterior derivative, where

$$
D \varphi=(d \varphi)_{\text {hor }}=d \varphi+[\omega \wedge \varphi],
$$

and where the wedge bracket $[\omega \wedge \varphi] \in C_{\mathrm{Ad}}^{\infty}\left(\Lambda^{1}(P) \otimes(\mathfrak{5})\right.$ is defined pointwise by $[\omega \wedge \varphi] \cdot Z=[\omega(Z), \varphi]$ for $Z \in \mathfrak{X}(P)$ (see Fischer [1985] for more on this terminology). Also, we let

$$
\Omega=D \omega=(d \omega)_{\mathrm{hor}}=d \omega+\frac{1}{2}[\omega \wedge \omega] \in A^{2}(P, \mathbf{6 5})
$$

denote the curvature 2-forms of $\omega$, where the wedge bracket

is given by $\quad[\omega \wedge \wedge] \in C_{\mathrm{Ad}}^{\infty}\left(\Lambda^{2}(P) \otimes(\mathfrak{5})\right.$

$$
[\omega \wedge \omega] \cdot\left(Z_{1}, Z_{2}\right)=\left[\omega\left(Z_{1}\right), \omega\left(Z_{2}\right)\right]-\left[\omega\left(Z_{2}\right), \omega\left(Z_{1}\right)\right]
$$

for $Z_{1}, Z_{2} \in \mathfrak{X}(P)$.

$$
=2\left[\omega\left(Z_{1}\right), \omega\left(Z_{2}\right)\right]
$$

For $F \in \operatorname{AUT}(P)$, and $\omega \in \mathscr{C}(P)$, we let $F^{*} \omega$ denote the pull-back of $\omega$ by $F$. Thus if $p \in P$ and $Z_{p} \in T_{p} P, F^{*} \omega$ is defined by

$$
F^{*} \omega(p) \cdot Z_{p}=\omega(F(p)) \cdot\left(T_{p} F \cdot Z_{p}\right) .
$$

An easy check shows that $F^{*} \omega \in \mathscr{C}(P)$, and indeed the map $F^{*}: \mathscr{C}(P) \rightarrow \mathscr{C}(P)$ is an affine transformation of $\mathscr{C}(P)$. Thus AUT $(P)$ acts on $\mathscr{C}(P)$ on the right by pull-back as a group of affine transformations,

$$
\mathscr{C}(P) \times \operatorname{Aut}(P) \rightarrow \mathscr{C}(P), \quad(\omega, F) \mapsto F^{*}(\omega .
$$

We shall be interested in the restricted action by $\operatorname{Aut}(P)$. Note that if $a \in Z(G)$, then $R_{a} \in \operatorname{Aut}(P)$ and $R_{a}^{*} \omega=\left(\operatorname{Ad}\left(a^{-1}\right) \cdot \omega=\omega\right.$, so that $Z_{G}(P) \subseteq I_{\omega}(P)$ for any connection $\omega$. Thus Aut $(P)$ does not act effectively on $\mathscr{C}(P)$. However since $Z_{G}(P)$ is a normal subgroup of $\operatorname{Aut}(P)$, this is easily remedied by considering the quotient group $\mathscr{A}(P)=Z_{G}(P) \backslash \operatorname{Aut}(P)$ of right cosets of $Z(G)$, and the induced action

$$
\mathscr{C}(P) \times \mathscr{A}(P) \rightarrow \mathscr{C}(P), \quad(\omega,[F]) \mapsto F^{*}(1),
$$

where $[F]=Z(G) \circ F$. Under the assumption that $P$ is a connected manifold, the resulting action is then effective (see Fischer [1985]).

Let $\theta \in C^{\infty}\left(\Lambda^{1}(G) \otimes(\mathfrak{b})\right.$ denote the Maurer-Cartan form on $G$; i.e., the unique left invariant Lie algebra valued 1 -form that satisfies

(1) $\theta(e)=\mathrm{id}_{\mathfrak{5}}:(\mathfrak{5} \rightarrow \mathbf{5}$, the identity transformation of $\mathbf{5}$.

(2) $\widetilde{L}_{a}^{*} \theta=\theta$ for all $a \in G$, where $\widetilde{L}_{a}: G \rightarrow G, b \mapsto a \cdot b$ denotes left translation on $G$. Thus $\theta$ is defined by

$$
\theta(a)=T_{a} \widetilde{L}_{a^{-1}}: T_{a} G \rightarrow \mathbf{6 5}, \quad v_{a} \mapsto T_{a} \tilde{L}_{a^{-1}} \cdot v_{a} .
$$

Note $\theta$ is now different from the canonical form $\theta$ discussed in the introduction. 
The following proposition is then a global version of the local transformation law for connection 1 -forms under a gauge transformation $u \in C_{\text {Aut }}^{\infty}(P, G)$ (see Kobayashi-Nomizu [1963], p. 66 or Bleecker [1981], p. 49).

1.3. Proposition. Let $\omega \in \mathscr{C}(P), F \in \operatorname{Aut}(P)$, and $u_{F} \in C_{\mathrm{Aut}}^{\infty}(P, G)$ correspond to $F$ as in Proposition 1.2. Let $\theta$ be the Maurer-Cartan form on $G$. Then

$$
F^{*} \omega=\operatorname{Ad}\left(u_{F}^{-1}\right) \cdot \omega+u_{F}^{*} \theta
$$

where $u_{F}^{-1}(p) \equiv\left(u_{F}(p)\right)^{-1}$.

Thus for $u \in C_{\mathrm{Aut}}^{\infty}(P, G)$, we define $u^{*}\left(\omega=\operatorname{Ad}\left(u^{-1}\right) \cdot \omega+u^{*} \theta\right.$, thereby generating the equation $F^{*} \omega=u_{F}^{*} \omega$.

There is another interesting interpretation of the equation $u^{*} \omega=\operatorname{Ad}\left(u^{-1}\right) \cdot \omega$ $+u^{*} \theta$. For $u \in C_{\text {Aut }}^{\infty}(P ; G)$, we would like to define a gauge covariant derivative of $u$. Since $u$ takes values in $G$, which in general is not a linear space, we must modify the usual definition of gauge covariant derivative. The ingredient needed is a means of identifying the tangent spaces of $G$ with 6 . This ingredient is provided by $\theta$. Thus a reasonable definition of the gauge covariant derivative $D u \in A^{1}(P,(\mathfrak{5})$ of $u$ is given by

$$
D u(p) \cdot Z_{p}=\theta(u(p)) \cdot\left(T_{p} u \cdot Z_{\mathrm{hol}}\right)=\left(u^{*} \theta\right)(p) \cdot Z_{\mathrm{hor}}=\left(u^{*} \theta\right)_{\mathrm{hor}} \cdot Z_{p},
$$

where $p \in P, Z_{p} \in T_{p} P$, and $Z_{\text {hor }}$ is the horizontal projection of $Z_{p}$. Thus $D u \equiv\left(u^{*} \theta\right)_{\text {hor }}$. A calculation as in Proposition 1.3 then shows that

$$
D u=\left(u^{*} \theta\right)_{\mathrm{hor}}=u^{*} \theta+\operatorname{Ad}\left(u^{-1}\right) \cdot \omega-\omega=u^{*} \omega-\omega .
$$

Thus in particular $u^{*} \omega=\omega$ if and only if $D u=0$.

For $T \in \mathfrak{X}_{G}^{v e r t}(P)$ and $\omega \in \mathscr{C}(P)$, let $L_{T} \omega \in A^{1}(P,(\mathfrak{5})$ denote the Lie derivative of $\omega$ with respect to $T$. That $L_{T} \omega$ is horizontal is the infinitesimal version of the fact that the difference between two connections is horizontal. Moreover, using the connection $\omega$, the bijection

$$
\mathfrak{X}_{G}^{\mathrm{vert}}(P) \rightarrow C_{\mathrm{Ad}}^{\infty}\left(P,(\mathfrak{H}), \quad T \mapsto \varphi_{T}\right.
$$

of Proposition 1.2 can be written as $T \mapsto \varphi_{T}=\omega(T)$, although this correspondence is independent of the connection. The following relationship between $L_{T}(1)$ and $D \varphi_{T}$ is of importance.

1.4. Lemma.Let $T \in \mathfrak{X}_{G}^{\mathrm{vert}}(P)$ and $\varphi_{T}=\omega(T) \in C_{\mathrm{Ad}}^{\infty}\left(P,(\mathfrak{5})\right.$. Then $L_{T}(1)=D \varphi_{T}$.

Proof. $L_{T} \omega=d i_{T} \omega+i_{T} d \omega=d \varphi_{T}+i_{T} d \omega$. Since $\Omega=D \omega$ is horizontal,

Hence

$$
\begin{aligned}
0=i_{T} D \omega & =i_{T}\left(d \omega+\frac{1}{2}[\omega \wedge \omega]\right)=i_{T} d \omega+\frac{1}{2}([\omega(T) \wedge \omega]-[\omega \wedge \omega(T)]) \\
& =i_{T} d \omega+\left[\varphi_{T} \wedge \omega\right] .
\end{aligned}
$$

$$
L_{T} \omega=d \varphi_{T}+i_{T} d \omega=d \varphi_{T}-\left[\varphi_{T} \wedge \omega\right]=d \varphi_{T}+\left[\omega \wedge \varphi_{T}\right]=D \varphi_{T} .
$$

For $\omega \in \mathscr{C}(P)$ and $p \in P$, let $\operatorname{Hol}_{p}(\omega) \subseteq G$ denote the holonomy group of $\omega$ with reference point $p \in P$. Then $\mathrm{Hol}_{p}(\omega)$ is a Lie subgroup of $G$ (Kobayashi-Nomizu [1963], p. 73), although it is not necessarily a closed subgroup. Note however that $\mathrm{Hol}_{p}(\omega)$ has at most a countable number of components (since $M$ is connected and 
paracompact), and thus $\operatorname{Hol}_{p}(\omega)$ satisfies the second axiom of countability as required by our definition of Lie group. Since $M$ is connected, all of the holonomy groups are conjugate in $G$ to each other, and we denote $\operatorname{Hol}(\omega)$ as any one of them.

For $p \in P$, we let $P(p)$ denote the holonomy bundle (or sub-bundle) through $p$, i.e., the set of points in $P$ that can be joined to $p$ by a horizontal curve. Then $P(p)$ $=Q\left(M, \operatorname{Hol}_{p}(\omega)\right)$ is a $\mathrm{Hol}_{p}(\omega)-\mathrm{PFB}$ over $M$, and the connection $\omega$ reduces to a connection on $Q\left(M, \mathrm{Hol}_{p}(\omega)\right)$ (Kobayashi-Nomizu [1963], p. 83).

Note that the holonomy sub-bundle $P(p)$ need only be an immersed subbundle. Although in general the closure of an immersed submanifold need not be a closed embedded submanifold, the closure $\overline{P(p)}$ of $P(p)$ will be a closed embedded submanifold in $P$, and in fact the closed sub-bundle $\overline{P(p)}=\overline{Q\left(M, \operatorname{Hol}_{p}(\omega)\right)}$ $=\overline{Q\left(M, \operatorname{Mol}_{p}(\omega)\right)}$ will be a reduced sub-bundle of $P(M, G)$ with structure group

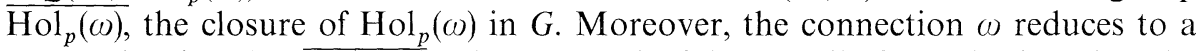
connection in $Q\left(M, \overline{\mathrm{Hol}}_{p}(\omega)\right)$. These remarks follow easily from the fact that the closure $H$ of a Lie subgroup $H$ of $G$ is a closed subgroup and hence an embedded submanifold.

In a similar vein as the above, we have the following lemma.

1.5. Lemma. Let $G$ be a topological group, $S$ a subset of $G$, and

$$
C_{G}(S)=\{a \in G \mid a \cdot s=s \cdot a \text { for all } s \in S\}
$$

the centralizer of $S$ in $G$. Then $C_{G}(S)$ is a closed subgroup of $G . A l s o, C_{G}(\bar{S})=C_{G}(S)$, where $\bar{S}$ denotes the closure of $S$ in $G$. Thus $C_{G}(S)=C_{G}(\bar{S})=\overline{C_{G}(S)}$, where $\overline{C_{G}(S)}$ denotes the closure of $C_{G}(S)$ in $G$.

Proof. Let $\left\{a_{n}\right\}$ be a sequence in $C_{G}(S)$ such that $a_{n} \rightarrow a \in \overline{C_{G}(S)}$. Then for all $n$ and $s \in S, a_{n} \cdot s=s \cdot a_{n}$, and so by continuity of the group operation, $a_{n} \cdot s \rightarrow a \cdot s$ and $s \cdot a_{n}$ $\rightarrow s \cdot a$. Thus $a \cdot s=s \cdot a$ and so $a \in C_{G}(S)$ and thus $C_{G}(S)$ is closed.

Similarly, let $\left\{s_{n}\right\}$ be a sequence in $S$ such that $s_{n} \rightarrow s \in \bar{S}$. Then for all $n$ and $a \in C_{G}(S), a \cdot s_{n}=s_{n} \cdot a$, and so as above $a \cdot s=s \cdot a$. Thus $a \in C_{G}(\bar{S})$, and so $C_{G}(S) \subseteq C_{G}(\bar{S})$. The reverse inclusion follows by definition of the centralizer.

Remark. In particular, the center of a topological group is closed.

We return now to $G$ being a Lie group.

\section{The Lie Group $I_{\omega}(P)$}

Let $\omega$ be a connection on the PFB $P(M, G)$. Let

$$
I_{\omega}(P)=\left\{F \in \operatorname{Aut}(P) \mid F^{*} \omega=\omega\right\}
$$

denote the internal symmetry group of $\omega$. We denote the corresponding subgroup of $C_{\text {Aut }}^{\infty}(P, G)$ as

$$
I_{\omega}(P, G)=\left\{u \in C_{\mathrm{Aut}}^{\infty}(P, G) \mid u^{*} \omega=\omega\right\},
$$

where $u^{*} \omega=\operatorname{Ad}\left(u^{-1}\right) \cdot \omega+u^{*} \theta$. Let

$$
\mathscr{I}_{\omega}(P)=\left(T \in \mathfrak{X}_{G}^{\mathrm{vert}}(P) \mid L_{T} \omega=0\right\}
$$

denote the Lie algebra of infinitesimal symmetries of $\omega$. That $\mathscr{I}_{\omega}(P)$ is a Lie algebra follows from the identity

$$
L_{\left[T_{1}, T_{2}\right]} \omega=L_{T_{1}} L_{T_{2}} \omega-L_{T_{2}} L_{T_{1}} \omega
$$


for $T_{1}, T_{2} \in \mathfrak{X}_{G}^{\text {vert }}$. Note that since infinitesimal symmetries are in $\mathfrak{X}_{G}^{\text {vert }}(P)$, they are automatically complete vector fields on $P$.

Since $L_{T} \omega=D \varphi_{T}$, the corresponding Lie algebra of $C_{\mathrm{Ad}}^{\infty}(P, \mathfrak{G})$ is

$$
\mathscr{I}_{\omega}\left(P,(\mathfrak{5})=\left\{\varphi \in C_{\mathrm{Ad}}^{\infty}(P,(\mathfrak{5}) \mid D \varphi=0\}=\operatorname{ker} D\right.\right.
$$

That $\mathscr{I}_{\omega}(P,(\mathfrak{5})$ is a Lie algebra follows from the identity

$$
D\left(\left[\varphi_{1}, \varphi_{2}\right]\right)=\left[D \varphi_{1} \wedge \varphi_{2}\right]+\left[\varphi_{1} \wedge D \varphi_{2}\right]
$$

for $\varphi_{1}, \varphi_{2} \in C_{\mathrm{Ad}}^{\infty}(P,(\mathfrak{5})$.

For $p \in P$, let $\operatorname{Hol}_{p}(\omega)$ be the holonomy group with reference point $p$, and let

$$
C_{G}\left(\operatorname{Hol}_{p}(\omega)\right)=\left\{a \in G \mid a \cdot b=b \cdot a \text { for all } b \in \operatorname{Hol}_{p}(\omega)\right\}
$$

denote the centralizer of $\operatorname{Hol}_{p}(\omega)$ in $G$. Although $\operatorname{Hol}_{p}(\omega)$ need not be a closed subgroup of $G$, by Lemma $1.5, C_{G}\left(\operatorname{Hol}_{p}(\omega)\right)$ is a closed subgroup of $G$, and hence a Lie subgroup. Its Lie algebra is

$$
C_{G}\left(\operatorname{Hol}_{p}(\omega)\right)=\left\{A \in \mathfrak{G} \mid \operatorname{Ad}(b) \cdot A=A \text { for all } b \in \operatorname{Hol}_{b}(\omega)\right\} .
$$

The following classical result will be our main workhorse for this section.

2.1. Proposition. Let $P(M, G)$ be a PFB, let $\omega \in \mathscr{C}(P)$, and let $u \in I_{\omega}(P, G)$. Then

(1) $u$ is constant on every holonomy sub-bundle $P(p)$;

(2) for $p \in P, u(p) \in C_{G}\left(\operatorname{Hol}_{p}(\omega)\right)$;

(3) $u$ is determined by its value at a single point. In particular, if $u\left(p_{0}\right)=e$ for some $p_{0} \in P$, then $u \equiv e$ identically on $P$.

Conversely if $u \in C_{\mathrm{Aut}}^{\infty}(P, G)$ is constant on a particular holonomy subbundle $P\left(p_{0}\right)$, then $u^{*} \omega=\omega$, and (1), (2), and (3) above hold.

Proof. (1): If $u \in I_{\omega}(P, G)$, then $u^{*} \omega=\operatorname{Ad}\left(u^{-1}\right) \cdot \omega+u^{*} \theta=\omega$. For $p \in P$ and $p_{1} \in P(p)$, let $\bar{c}(\lambda), \lambda \in[0,1]$, denote a horizontal curve from $p$ to $p_{1}$. Then $\omega(\bar{c}(\lambda)) \cdot \bar{c}(\lambda)=0$ for $\lambda \in[0,1]$, and so $u^{*} \theta(\bar{c}(\lambda)) \cdot \bar{c}^{\prime}(\lambda)=0$. Let $\lambda \mapsto a(\lambda)=(u \circ \bar{c})(\lambda)$, a curve in $G$. Then

Since

$$
\begin{aligned}
u^{*} \theta(\bar{c}(\lambda)) \cdot \bar{c}^{\prime}(\lambda) & =\theta\left(u(\bar{c}(\lambda)) \cdot T_{\bar{c}(\lambda)} u \cdot \bar{c}^{\prime}(\lambda)=\theta(u \circ \bar{c})(\lambda)\right) \cdot(u \circ \bar{c})^{\prime}(\lambda) \\
& =\theta(a(\lambda)) \cdot a^{\prime}(\lambda)=0 .
\end{aligned}
$$

$$
\theta(a(\lambda))=T_{a(\lambda)} \widetilde{L}_{(a(\lambda))^{-1}}: T_{a(\lambda)} G \rightarrow(\mathfrak{H}
$$

is an isomorphism, $a^{\prime}(\lambda)=(u \circ \bar{c})^{\prime}(\lambda)=0$. Thus $(u \circ \overline{\mathcal{c}})(\lambda)$ is constant and so $u\left(p_{1}\right)$ $=u(p)$.

(2): For any $a \in \mathrm{Hol}_{p}(\omega), p \cdot a \in P(p)$, and since $u$ is constant on $P(p), u(p)$ $=u(p \cdot a)=a^{-1} \cdot u(p) \cdot a$. Thus $u(p) \in C_{G}\left(\operatorname{Hol}_{p}(\omega)\right)$.

(3): If $u_{1} \in I_{\omega}(p)$ and $u_{1}\left(p_{0}\right)=u\left(p_{0}\right)$ for some $p_{0} \in P$, then by (1), $u_{1}$ is constant on $P\left(p_{0}\right)$, and hence $u$ and $u_{1}$ agree on $P\left(p_{0}\right)$. Since $P\left(p_{0}\right)$ intersects each fiber of $P$ at least once, $u_{1}$ agrees with $u$ on at least one point in each fiber, and thus by their transformation properties, on the entire fiber. Thus $u_{1}=u$ on $P$.

Conversely, if $u \in C_{\mathrm{Aut}}^{\infty}(P, G)$ is constant on a particular holonomy subbundle $P\left(p_{0}\right)$, then since $P\left(p_{0} \cdot a\right)=P\left(p_{0}\right) \cdot a$,

$$
u\left(P\left(p_{0} \cdot a\right)\right)=u\left(P\left(p_{0}\right) \cdot a\right)=a^{-1} \cdot u\left(P\left(p_{0}\right)\right) \cdot a,
$$


and so $u$ is constant on every holonomy sub-bundle. Thus for $p \in P$, let $\bar{X} \in H_{p} \subseteq T_{p} P$ be a vector at $p$ which is horizontal with respect to the connection ( $)$, so that $\omega(p) \cdot \bar{X}=0$. Thus

$$
\left(u^{*}(\omega)(p) \cdot \bar{X}=\operatorname{Ad}\left(u^{-1}(p)\right) \cdot(\omega(p) \cdot \bar{X})+u^{*} \theta(p) \cdot \bar{X}=\theta(u(p)) \cdot\left(T_{p} u \cdot \bar{X}\right) .\right.
$$

By the Holonomy Reduction Theorem (Kobayashi-Nomizu [1963], p. 83), the connection $\omega$ on $P$ reduces to a connection on $P(p)$. Thus the horizontal space $H_{p}$ is contained in the tangent space $T_{p}(P(p))$. Since $u$ is constant on $P(p), T_{p} u \cdot \bar{X}=0$. Thus $\left(u^{*} \omega\right)(p) \cdot \bar{X}=0$, and so $H_{p}=\operatorname{ker} \omega(p) \leqq \operatorname{ker}\left(u^{*} \omega\right)(p)$. Since $u^{*} \omega$ is also a connection on $P$, dim $\operatorname{ker} \omega(p)=\operatorname{dim} \operatorname{ker}\left(u^{*} \omega\right)(p)$, and so $\operatorname{ker} \omega(p)=\operatorname{ker}\left(u^{*} \omega\right)(p)$. Thus $\omega(p)$ and $\left(u^{*} \omega\right)(p)$ have the same horizontal subspace at $p$, and since $p$ was arbitrary, $u^{*} \omega=\omega$ ). Note that all connections determine the same vertical subspace $V_{p}=\operatorname{ker} T_{p} \pi$, reflected by the requirement that $\omega\left(A^{*}\right)=A$ for all $A \in \mathfrak{G}$.

Remarks. 1. Thus Proposition 2.1 can be summarized by

$I_{\omega}(P, G)=\left\{u \in C_{\mathrm{Aut}}^{\infty}(P, G) \mid u\right.$ is constant on the holonomy sub-bundles of $\left.P\right\}$.

2. Property (3) can be rephrased in terms of $I_{\omega}(P)$ as follows:

2.1.1. Proposition. If $F \in I_{\omega}(P)$ fixes a point $p_{0} \in P, F\left(p_{0}\right)=p_{0}$, then $F=\mathrm{id}$, the identity automorphism of $P$.

3. As we have seen in Sect. $1, D u=\left(u^{*} \theta\right)_{\text {hor }}=u^{*} \omega-\omega$. Thus $u \in I_{\omega}(P, G)$ if and only if $D u=0$ if and only if $u$ is a gauge covariant constant if an only if (1), (2), and (3) above hold.

The infinitesimal version of Proposition 2.1 is the following.

2.2. Proposition. Let $P(M, G)$ be a $\mathrm{PFB}$, let $\varphi \in \mathscr{I}_{\omega}(P,(\mathfrak{5})=\operatorname{ker} D$. Then

(1) $\varphi$ is constant on every holonomy sub-bundle $P(p)$;

(2) for $p \in P, \varphi(p) \in c_{G}\left(\operatorname{Hol}_{p}(\omega)\right)=\left\{A \in \mathfrak{G} \mid \operatorname{Ad}(b) \cdot A=A\right.$ for all $\left.b \in \operatorname{Hol}_{p}(\omega)\right\}$;

(3) $\varphi$ is determined by its value at a single point. In particular, if $\varphi\left(p_{0}\right)=0$ for some $p_{0} \in P$, then $\varphi \equiv 0$ on $P$.

Conversely, if $\varphi \in C_{\mathrm{Ad}}^{\infty}(P,(\mathfrak{5})$ is constant on a particular holonomy subbundle, then $D \varphi=0$, and (1), (2), and (3) above hold.

Proof. If $D \varphi=(d \varphi)_{\text {hor }}=d \varphi+[\omega, \varphi]=0$, then on a horizontal curve $\bar{c}(\lambda)$,

$$
D \varphi(\bar{c}(\lambda)) \cdot \bar{c}^{\prime}(\lambda)=d \varphi(\bar{c}(\lambda)) \cdot \bar{c}^{\prime}(\lambda)=(\varphi \circ \bar{c})^{\prime}(\lambda)=0 .
$$

Thus $\varphi$ is constant on horizontal curves and hence is constant on every holonomy sub-bundle. The proof now proceeds as in Proposition 2.1. Alternately, the proposition can be proven by taking curves in $I_{\omega}(P, G)$ and using Proposition 2.1 .

Remark. In terms of $\mathscr{I}_{\omega}(P)=\left\{T \in \mathfrak{X}_{G}^{\mathrm{vert}}(P) \mid L_{T} \omega=0\right\}$, property (3) can be rephrased as

2.2.1. Proposition. If $T \in \mathscr{I}_{\omega}(P)$ satisfies $T\left(p_{0}\right)=0$ for some $p_{0} \in P$, then $T \equiv 0$.

Using Proposition 2.1, we can now derive a "formula" for $I_{\omega}(P, G)$. 
2.3. Theorem. Let $P(M, G)$ be a PFB, let $\omega \in \mathscr{C}(P)$, and let $p_{0} \in P$. Then the evaluation map at $p_{0}$,

$$
\operatorname{eval}_{p_{0}}: I_{\omega}(P, G) \rightarrow C_{G}\left(\operatorname{Hol}_{p_{0}}(\omega)\right), \quad u \mapsto u\left(p_{0}\right),
$$

is a group isomorphism. Similarly, the evaluation map

$$
\operatorname{eval}_{p_{0}}^{\prime}: \mathscr{I}_{\omega}(P, G) \rightarrow c_{G}\left(\operatorname{Hol}_{p_{0}}(\omega)\right), \quad \varphi \mapsto \varphi\left(p_{0}\right)
$$

is a Lie algebra isomorphism.

Proof. By the definition of the group structure in $C_{\text {Aut }}^{\infty}(P, G)$,

$$
\operatorname{eval}_{p_{0}}\left(u_{1} \cdot u_{2}\right)=u_{1}\left(p_{0}\right) \cdot u_{2}\left(p_{0}\right)=\left(\operatorname{eval}_{p_{0}}\left(u_{1}\right)\right) \cdot\left(\operatorname{eval}_{p_{0}}\left(u_{2}\right)\right)
$$

and so eval $p_{0}$ is a group homomorphism. It is injective by property (3) of Proposition 2.1. that

To show surjectivity, let $a \in C_{G}\left(\operatorname{Hol}_{p_{0}}(\omega)\right)$, and define a function $u: P \rightarrow G$ such

$$
u(p)=\left\{\begin{array}{lll}
a & \text { if } & p \in P\left(p_{0}\right) \\
b^{-1} \cdot a \cdot b & \text { if } & p \in P\left(p_{0} \cdot b\right)
\end{array}\right.
$$

$u$ is well-defined, for if $p \in P\left(p_{0}\right) \cap P\left(p_{0} \cdot b\right)$, then $b \in \operatorname{Hol}_{p_{0}}(\omega)$ [and $P\left(p_{0}\right)=P\left(p_{0} \cdot b\right)$ ], and thus $b^{-1} \cdot a \cdot b=a$ since $a \in C_{G}\left(\operatorname{Hol}_{p_{0}}(\omega)\right)$. To find the transformation properties of $u$, let $p \in P\left(p_{0} \cdot b\right)$, so $u(p)=b^{-1} \cdot a \cdot b$. Then for $c \in G$,

$$
p \cdot c \in P\left(p_{0} \cdot b\right) \cdot c=P\left(p_{0} \cdot b \cdot c\right),
$$

So

$$
u(p \cdot c)=(b \cdot c)^{-1} \cdot a \cdot(b \cdot c)=c^{-1} \cdot\left(b^{-1} \cdot a \cdot b\right) \cdot c=c^{-1} \cdot u(p) \cdot c .
$$

If $\mathrm{Hol}_{p_{0}}(\omega)$ is a closed subgroup of $G, P\left(p_{0}\right)$ is an embedded submanifold, and since by construction $u$ is constant on the holonomy sub-bundles, it follows that $u$ is a $C^{\infty}$ function on $P$. Thus $u \in C_{\text {Aut }}^{\infty}(P, G)$, and by Proposition $2.1, u^{*} \omega=\omega$.

If $\mathrm{Hol}_{p_{0}}(\omega)$ is not closed in $G$, then by Lemma $1.5, C_{G}\left(\operatorname{Hol}_{p}(\omega)\right)=C_{G} \overline{\left(\operatorname{Hol}_{p_{0}}(\omega)\right)}$. Thus if $a$ centralizes $\mathrm{Hol}_{p_{0}}(\omega), a$ centralizes $\overline{\operatorname{Hol}_{p_{0}}(\omega)}$. Thus $u$ defined above is constant on the embedded submanifold $\overline{P\left(p_{0}\right)}=Q\left(M, \overline{\left.\text { Hol }_{p_{0}}(\omega)\right)}\right.$. Thus, as above, $u$ is a $C^{\infty}$ function on $P, u \in C_{\text {Aut }}^{\infty}(P, G)$, and as $u$ is constant on the holonomy subbundles, $u^{*}(\omega)=\omega$.

Remark. Another approach to the surjectivity of eval $p_{0}$ that avoids the complication of non-closed holonomy groups is as follows. Let $x_{0}=\pi\left(p_{0}\right)$, and let $\mathrm{Hol}_{x_{0}}$ denote the holonomy group with reference point $x_{0}$. Then $\mathrm{Hol}_{x_{0}}$ is a Lie subgroup of $G_{x_{0}}=\left(G_{\mathrm{Aut}}(M)\right)_{x_{0}}$, the group of automorphisms of the fiber $P_{x_{0}}$. If $a \in C_{G}\left(\mathrm{Hol}_{p_{0}}\right)$, let $a_{x_{0}} \in G_{x_{0}}$ be the corresponding element in $G_{x_{0}}$. Then $a_{x_{0}} \in C_{G_{x_{0}}}\left(\mathrm{Hol}_{x_{0}}\right)$. We construct a smooth section $u_{M} \in C^{\infty}\left(G_{\mathrm{Aut}}(M)\right)$ as follows. If $x_{1} \in M$, let $c:[0,1] \rightarrow M$, $\lambda \rightarrow c(\lambda)$, be a smooth curve from $x_{0}$ to $x_{1}$, let $\bar{c}^{-1}(\lambda)=c(1-\lambda)$, let $\tau_{c}: P_{x_{0}} \rightarrow P_{x_{1}}$ denote parallel translation of the fiber $P_{x_{0}}$ along $c$, and let $\tau_{c}^{-1}: P_{x_{1}} \rightarrow P_{x_{0}}$ denote its inverse. Define $u_{M}\left(x_{1}\right)$ by

$$
u_{M}\left(x_{1}\right)=\tau_{c} \circ a_{x_{0}} \circ \tau_{c}^{-1}
$$


Then $u_{M}\left(x_{1}\right)$ is independent of the curve $c$ from $x_{0}$ to $x_{1}$ since if $c_{1}$ and $c_{2}$ are two such curves,

$$
\begin{aligned}
\tau_{c_{1}} \circ a_{x_{0}} \circ \tau_{c_{1}-1} & =\tau_{c_{2}} \circ\left(\tau_{c_{2}-1} \circ \tau_{c_{1}}\right) \circ a_{x_{0}} \circ\left(\tau_{c_{1}^{-1}} \circ \tau_{c_{2}}\right) \circ \tau_{c_{\overline{2}^{-1}}} \\
& =\tau_{c_{2}} \circ \tau_{\gamma} \circ a_{x_{0}} \circ \tau_{\gamma}^{-1} \circ \tau_{c_{2}-1}=\tau_{c_{2}} \circ a_{x_{0}} \circ \tau_{c_{2}-1},
\end{aligned}
$$

where $\gamma=c_{1} \cdot c_{2}^{-1}$ is the closed composite curve based at $x_{0}$, and the last equality follows since $\tau_{\gamma} \in \mathrm{Hol}_{x_{0}}$ and $a_{x_{0}} \in C_{G_{x_{0}}}\left(\mathrm{Hol}_{x_{0}}\right)$. Let $u \in C_{\text {Aut }}^{\infty}(P, G)$ correspond to $u_{M} \in C^{\infty}\left(G_{\text {Aut }}(M)\right)$. Then by construction of $u_{M}, u\left(p_{0}\right)=a$ and $u$ is constant on the holonomy sub-bundles of $P$, and so $u^{*}(l)=\omega$.

By Lemma 1.5, $C_{G}\left(\mathrm{Hol}_{p_{0}}(\omega)\right)$ is a closed subgroup of $G$, and hence a closed Lie subgroup. In particular, $C_{G}\left(\mathrm{Hol}_{p_{0}}(\omega)\right)$ is an embedded submanifold in $G$. We put a Lie group structure on $I_{\omega}(P, G)$ by declaring that the group isomorphism

$$
\operatorname{eval}_{p_{0}}: I_{\omega}(P, G) \rightarrow C_{G}\left(\mathrm{Hol}_{p_{0}}(\omega)\right)
$$

is a Lie group isomorphism. With respect to this Lie group structure,

$$
\operatorname{eval}_{p_{0}}^{\prime}=T_{e} \operatorname{eval}_{p_{0}}: \mathscr{I}_{\omega}\left(P,(\mathfrak{5}) \rightarrow c_{G}\left(\operatorname{Hol}_{p_{0}}(\omega)\right)\right.
$$

is the induced Lie algebra isomorphism.

Clearly, for different $p \in P$, the Lie group structures induced on $I_{\omega}(P, G)$ are all isomorphic. Moreover, any other second countable Lie group structure on $I_{\omega}(P, G)$ must be isomorphic to that induced by eval $p_{0}$ (see e.g., Helgason [1962], p. 109, or Warner [1971], p. 95). Thus the Lie group structure on $I_{\omega}(P, G)$ is unique (up to isomorphism).

Using the group bijection between $I_{\omega}(P)$ and $I_{\omega}(P, G)$, we carry the Lie group structure of $I_{\omega}(P, G)$ back to $I_{\omega}(P)$. With respect to this structure, the map

$$
I_{\omega}(P) \rightarrow C_{G}\left(\operatorname{Hol}_{p_{0}}(\omega)\right), \quad F \mapsto u_{F}\left(p_{0}\right)
$$

is a Lie group isomorphism, where $u_{F}$ corresponds to $F$ by Proposition 1.1. Thus we have the following "formula" for $I_{\omega}(P), I_{\omega}(P) \approx C_{G}(\operatorname{Hol}(\omega))$, where $\operatorname{Hol}(\omega)$ denotes any one of the holonomy groups $\operatorname{Hol}_{p}(\omega), p \in P$.

Remark. If $a \in C_{G}\left(\mathrm{Hol}_{p_{0}}(\omega)\right), p_{0} \in P, F \in I_{\omega}(P)$ is determined by the equation $F\left(p_{0}\right)$ $=p_{0} \cdot a$ [since $F \in I_{\omega}(P), F$ is uniquely determined by its value at a point]. Note, however, that although $R_{a}$ solves this equation, $R_{a}$ is not the corresponding internal symmetry of $\omega$ unless $a \in Z(G)$, and then $R_{a} \in \operatorname{Aut}(P)$. Thus $I_{\omega}(P)$, although isomorphic to the subgroup $C_{G}\left(\mathrm{Hol}_{p_{0}}(\omega)\right)$ of $G$, does not in general have a representation as a subgroup of $G(P)=\left\{R_{a} \mid a \in G\right\}$. Indeed $I_{\omega}(P)$ is a subgroup of $\operatorname{Aut}(P)$, and $G(P)$ is a subgroup of $\operatorname{Diff}(P)$.

\section{Some Examples}

We now give some examples of the formula $I_{\omega}(P) \approx C_{G}(\operatorname{Hol}(\omega))$.

3.1. Example. Let $P(M, G)$ be a PFB with Abelian structure group $G$. Then $Z(G)=G$, and so for all $a \in G, R_{a} \in \operatorname{Aut}(P)$. Let $\omega \in \mathscr{C}(P)$ with holonomy group $\operatorname{Hol}(\omega) \subseteq G$. Note that since the holonomy group of $\omega$ with respect to different 
points are all conjugate to each other in $G, \operatorname{Hol}(\omega)$ is independent of reference point.

Since $G$ is abelian, $C_{G}(\operatorname{Hol}(\omega))=G$, and so $I_{\omega}(P) \approx G$. But since for all $a \in G$, $R_{a} \in \operatorname{Aut}(P)$, and $R_{a}^{*} \omega=\omega,\left\{R_{a} \mid a \in G\right\} \cong I_{\omega}(P)$, so that $I_{\omega}(P)=G(P)=\left\{R_{a} \mid a \in G\right\} \approx G$. Thus in the Abelian case, for any connection $\omega, I_{\omega}(P)$ is actually isomorphic to the structure group of the bundle. The Lie algebra of $I_{\omega}(P)$ is $\mathscr{I}_{\omega}(P)=\left\{A^{*} \mid A \in \mathbb{6}\right\}$ $=6 \mathfrak{5}(P) \approx 05$. Similarly,

$$
I_{\omega}(P, G)=\{u: P \rightarrow G \mid u \text { is constant on } P\}
$$

with Lie algebra

$$
\mathscr{I}_{\omega}(P, \mathfrak{G})=\{\varphi: P \rightarrow \mathfrak{G} \mid \varphi \text { is constant on } P\} .
$$

In physical field theories, the Abelian case is of interest inasmuch as electromagnetism can be formulated in terms of a connection $\omega$ on a U(1)-PFB $P(M, \mathrm{U}(1))$ over a Lorentz manifold $(M, g)$. For any such connection the internal symmetry group is then given by $I_{\omega}(P) \approx \mathrm{U}(1)$. Since $I_{\omega}(P)$ leads to global conservation laws, in this case (or more generally, in any Abelian case), the structure group U(1) gives rise to global conservation laws for the resulting field theory (see also Sect. 4), and Fischer [1985]).

3.2. Example. Let $P(M, G)=M \times G$ be the product bundle and let $\omega_{0}$ be the canonical flat connection on $P$. Thus if $\pi_{2}: M \times G \rightarrow G,(x, a) \mapsto a$, denotes projection onto the second factor, then $\omega_{0}=\pi_{2}^{*} \theta$, where $\theta$ is the Maurer-Cartan form on $G$. Then $\operatorname{Hol}\left(\omega_{0}\right)=\{e\}$ (again independent of reference point), and $C_{G}\left(\operatorname{Hol}\left(\omega_{0}\right)\right)=C_{G}(\{e\})=G$, so that $I_{\omega_{0}}(P) \approx G$ is again isomorphic to the structure group of the bundle. In this case however, $I_{\omega_{0}}(P)$ is not equal to $G(P)$, since the right translations $R_{a}: P \rightarrow P$ are not automorphisms of the bundle [unless $a \in Z(G)$ ]. However, on a product bundle, $G$ can be represented as a group of left translations

$$
a \mapsto L_{a}=\left(\mathrm{id}_{M} \times \tilde{L}_{a}\right): M \times G \rightarrow M \times G, \quad(x, b) \mapsto(x, a b),
$$

where $\widetilde{L}_{a}$ is left translation on $G$ by $a$, and this action of $G$ on $M \times G$ commutes with the right action of $G$ on $M \times G$,

$$
L_{a} \circ R_{b}(x, c)=(x, a c b)=R_{b} \circ L_{a}(x, c) .
$$

Thus for all $a \in G, L_{a} \in \operatorname{Aut}(P)$, and also

$$
L_{a}^{*} \omega_{0}=L_{a}^{*} \pi_{2}^{*} \theta=\left(\pi_{2} \circ L_{a}\right)^{*} \theta=\left(\tilde{L}_{a} \circ \pi_{2}\right)^{*} \theta=\pi_{2}^{*} \tilde{L}_{a}^{*} \theta=\pi_{2}^{*} \theta=\omega_{0},
$$

since $\theta$ is invariant by left translations. Hence $L_{a} \in I_{\omega_{0}}(P)$. Thus $I_{\omega_{0}}(P)$ can be displayed concretely as the group of left translations of $M \times G$,

$$
I_{\omega_{0}}(M \times G)=\left\{L_{a}: M \times G \rightarrow M \times G \mid a \in G\right\}=G_{L},
$$

where $G_{L}$ is defined only for product bundles, or for bundles isomorphic to product bundles.

The Lie algebra of $G_{L}$ is given by

$$
\mathfrak{G}_{L}=\left\{(0, \tilde{A}) \in \mathfrak{X}_{G}^{\mathrm{vert}}(M \times G) \mid A \in(\mathfrak{G}\},\right.
$$

where $\tilde{A}$ is the left-invariant vector field on $G$ corresponding to $A \in \mathfrak{G}$. Thus for $a \in G, \tilde{A}(a)=T_{e} \tilde{L}_{a} \cdot A$. 
3.3. Example. As a slightly more general example, let $\omega$ be a connection on a PFB $P(M, G)$ whose holonomy group $\operatorname{Hol}(\omega)=\{e\}$. Then for $p_{0} \in P$, the holonomy bundle $P\left(p_{0}\right)$ is an $\{e\}$-PFB over $M$, or a global cross-section of $P(M, G)$. Thus $P(M, G)$ is bundle isomorphic to the product bundle $M \times G$ (but not canonically) and $\omega$ is isomorphic to the canonical flat connection $\omega_{0}$ on $M \times G$.

Then as above $I_{\omega}(P) \approx G$, and using an isomorphism of $P(M, G)$ with $M \times G$, $I_{\omega}(P)$ can be represented as a group of "left translations" on $P(M, G)$.

As a particular subcase, if $M$ is simply connected and $\omega$ is a flat connection on $P(M, G)$, then $\operatorname{Hol}(\omega)=\{e\}$, and so $I_{\omega}(P) \approx G$. This case is of interest for Yang-Mills field theories over a simply connected spacetime $M$, often taken to be either $\mathbb{R}^{4}$ or $S^{4}$. In this case, if the connection $\omega$ on $P(M, G)$ is flat, then $I_{\omega}(P) \approx G$. Ignoring the distinction between left and right translations, the structure group $G$ is said to induce the global conservation laws of the associated Lagrangian field theory (see also Sect. 4).

We now give some examples where we must compute the centralizer $C_{G}(\operatorname{Hol}(\omega))$.

3.4. Example. Let $M=S^{n}$ taken with its usual metric $g_{0}$ and orientation as the unit sphere in $\mathbb{R}^{n+1}$. Let $L\left(S^{n}\right)$ denote its frame bundle, and let $\omega_{0}$ be the Levi-Civita connection of $g_{0}$ on $L\left(S^{n}\right)$. Then $\operatorname{Hol}\left(\omega_{0}\right)=\mathrm{SO}(n)$ (see Poor [1981], p. 64, for the calculation), so that

$$
I_{\omega_{0}}\left(L\left(S^{n}\right)\right) \approx C_{G L(n)}(\mathrm{SO}(n)) .
$$

Let $\mathbb{R}^{*}=\mathbb{R}-\{0\}$. Then we have the following.

3.4.1. Lemma. For $n \in Z^{+}, n \neq 2$,

For $n=2$,

$$
C_{G L(n)}(\mathrm{SO}(n))=Z(G L(n))=\left\{\lambda I \mid \lambda \in \mathbb{R}^{*}\right\} \approx \mathbb{R}^{*} .
$$

$$
C_{G L(2)}(\mathrm{SO}(2))=\mathbb{R}^{*} \cdot \mathrm{SO}(2)=\left\{\lambda \cdot a \mid \lambda \in \mathbb{R}^{*} \text { and } a \in \mathrm{SO}(2)\right\} .
$$

Proof. Since $\mathrm{SO}(n)$ acts irreducibly on $\mathbb{R}^{n}$, if $A \in G L(n)$ commutes with every element of $\mathrm{SO}(n)$, then by Schur's lemma (see e.g., Kobayashi-Nomizu [1969], p. 277), if $n$ is odd, then $A=\lambda I, \lambda \in \mathbb{R}^{*}$, or if $n=2 m$ is even,

$$
A=\left(\begin{array}{cc}
a I_{m} & b I_{m} \\
-b I_{m} & a I_{m}
\end{array}\right), \quad a^{2}+b^{2} \neq 0 .
$$

Since for all $n \in Z^{+}, Z(G L(n)) \subseteq C_{G L(n)}(\mathrm{SO}(n))$, for $n$ odd, we have equality. If $n$ is even, let $B \in \operatorname{SO}(2 n)$. Then a conjugate of $B$ can be put into the standard form

$$
B\left(\theta_{1}, \ldots, \theta_{m}\right)=\left(\begin{array}{ccc}
B\left(\theta_{1}\right) & & \\
& \ddots & \\
& & B\left(\theta_{m}\right)
\end{array}\right)
$$

where $B\left(\theta_{i}\right)=\left(\begin{array}{cc}\cos \theta_{i} & \sin \theta_{n} \\ -\sin \theta_{i} & \cos \theta_{i}\end{array}\right)$. A matrix computation shows that if $m \geqq 2$ and $A$ above commutes with $B\left(\theta_{1}, \ldots, \theta_{m}\right)$ for all $\theta_{i}$, then $b=0$. Hence in this case $Z(G L(n))$ $=C_{G L(n)}(\mathrm{SO}(n))$. If $n=2$, all elements $A$ of the above form commute with $\mathrm{SO}(2)$, so 
that

$$
C_{G L(2)}(\mathrm{SO}(2))=\mathbb{R}^{+} \cdot \mathrm{SO}(2)=\mathbb{R}^{*} \cdot \mathrm{SO}(2)
$$

For $\lambda \in \mathbb{R}^{*}$, denote by $\lambda$ also the map

$$
\lambda: L\left(S^{n}\right) \rightarrow L\left(S^{n}\right), \quad p \mapsto \lambda p,
$$

where if $p=\left(X_{1}, \ldots, X_{n}\right)$ is a frame at $x, \lambda p=\left(\lambda X_{1}, \ldots, \lambda X_{n}\right)$ is also a frame at $x$. Thus for $n \neq 2$,

$$
I_{\omega_{0}}\left(L\left(S^{n}\right)\right)=\left\{\lambda: L\left(S^{n}\right) \rightarrow L\left(S^{n}\right) \mid \lambda \in \mathbb{R}^{*}\right\} \approx \mathbb{R}^{*},
$$

with a similar result for $n=2$.

Now let $0^{+}\left(S^{n}\right) \subseteq L\left(S^{n}\right)$ denote the $\mathrm{SO}(n)$ bundle of positively oriented orthonormal frames on $S^{n}$, and let $\omega_{0}^{\prime}$ denote the Levi-Civita connection of $g_{0}$ on $0^{+}\left(S^{n}\right)$. Then $\omega_{0}^{\prime}$ is the reduction of $\omega_{0}$ to $0^{+}\left(S^{n}\right)$, and so $\operatorname{Hol}\left(\omega_{0}^{\prime}\right)=\operatorname{Hol}\left(\omega_{0}\right)=\operatorname{SO}(n)$. Thus

$$
I_{\omega_{0}^{\prime}}\left(0^{+}\left(S^{n}\right)\right) \approx C_{\mathrm{SO}(n)}(\mathrm{SO}(n))=Z(\mathrm{SO}(n))=\left\{\begin{array}{ll}
\{I\} & \text { for } n \text { odd } \\
\{ \pm I\} & \text { for } n \text { even } \geqq 4 \\
\mathrm{SO}(2) \text { for } n=2
\end{array} \square\right.
$$

Remark. The above example shows that the internal symmetry group of a connection can change when the connection is reduced to a sub-bundle (although it need not necessarily change; see Example 3.6). For comparison's sake, note that the classical affine group $A_{\omega}(M)$ is independent of any reduction of $\omega$ to a subbundle. In the case at hand, for $n \geqq 2$,

$$
A_{\omega_{0}}\left(S^{n}\right)=A_{\omega_{0}^{\prime}}\left(S^{n}\right)=I_{g_{0}}\left(S^{n}\right)=O(n+1) .
$$

Also, we remark that $A_{\omega_{0}}\left(S^{n}\right) \subseteq \operatorname{Diff}\left(S^{n}\right)$, whereas $I_{\omega_{0}}\left(L\left(S^{n}\right)\right) \subseteq \operatorname{Aut}\left(L\left(S^{n}\right)\right)$, so that

$$
\operatorname{AUT}_{\left(\theta, \omega_{0}\right)}\left(L\left(S^{n}\right)\right) \cap I_{\omega_{0}}\left(L\left(S^{n}\right)\right)=\left\{\operatorname{id}_{L\left(S^{n}\right)}\right\},
$$

where $\theta$ is here the canonical 1 -form on $L\left(S^{n}\right)$.

3.5. Example. Let $P(M, G)$ be a PFB with $\operatorname{dim} M \geqq 2$, let $H$ be a Lie subgroup of $G$, and let $i: Q(M, H) \rightarrow P(M, G)$ be a reduction of $G$ to $H$. Assume that $Q$ is a connected manifold. Then there exists a connection $\omega_{0}$ on $Q(M, H)$ such that $\operatorname{Hol}\left(\omega_{0}\right)=H$ (see Kobayashi-Nomizu [1963], p. 90). Also $\omega_{0}$ induces a unique connection $\omega_{1}$ on $P(M, G)$ such that $\omega_{0}=i^{*} \omega_{1}$ and $\operatorname{Hol}\left(\omega_{1}\right)=\operatorname{Hol}\left(\omega_{0}\right)=H$. Now

$$
I_{\omega_{0}}(Q) \approx C_{H}\left(\operatorname{Hol}\left(\omega_{0}\right)\right)=C_{H}(H)=Z(H)
$$

and

$$
I_{\omega_{1}}(P) \approx C_{G}\left(\operatorname{Hol}\left(\omega_{0}\right)\right)=C_{G}(H)
$$

We give two special cases of this example.

3.6. Example. Let $M$ be a $2 m$-dimensional orientable manifold, $m \geqq 1, J$ an almost complex structure on $M\left(J^{2}=-I\right)$, and $g$ a Hermitian metric on $M, g(J X, J Y)$ $=g(X, Y)$. Consider $M$ with the natural orientation induced by $J$ and let $C(M)=P(M, G L(m, \mathbb{C}))$ denote the complex linear frame bundle; 
$0^{+}(M)=P(M, \mathrm{SO}(2 m))$ the bundle of oriented orthonormal frames and $U(M)=C(M) \cap 0^{+}(M)=P(M, U(m))$ the unitary frame bundle.

In the real representation of $G l(m, \mathbb{C})$,

$$
G L(m, \mathbb{C}) \rightarrow G L(2 m, \mathbb{R}), \quad A+i B \mapsto\left(\begin{array}{cc}
A & B \\
-B & A
\end{array}\right),
$$

where $A$ and $B$ are real $m \times m$ matrices,

$$
U(m)=G L(m, \mathbb{C}) \cap O(2 m)=G L(m, \mathbb{C}) \cap \mathrm{SO}(2 m),
$$

the latter equation following from the fact that $G L(m, \mathbb{C})$ is connected and hence lies in $G L^{0}(2 m, \mathbb{R})$, the connected component of the identity of $G l(m, \mathbb{R})$.

Let $i: U(M) \rightarrow 0^{+}(M)$ denote the bundle reduction of $0^{+}(M)$ to $U(M)$ induced by the almost complex structure $J$. Since $M$ is connected and since $\mathrm{U}(m)$ is connected ( $m \geqq 1$ ), it follows that the total space $\mathrm{U}(M)$ is connected. Also, $\operatorname{dim} M=2 m \geqq 2$, and so there exists a connection $\omega_{0}$ on $\mathrm{U}(M)$ such that $\operatorname{Hol}\left(\omega_{0}\right)=\mathrm{U}(m)$. This connection then induces a unique connection $\omega_{1}$ on $0^{+}(M)$ such that $\omega_{0}=i^{*} \omega_{1}$, and such that $\operatorname{Hol}\left(\omega_{0}\right)=\operatorname{Hol}\left(\omega_{1}\right)=\mathrm{U}(m)$. For these connections,

$$
I_{\omega_{0}}(\mathrm{U}(M)) \approx C_{\mathrm{U}(m)}(\mathrm{U}(m))=Z(\mathrm{U}(m))=\left\{e^{i \theta} I_{m} \mid \theta \in \mathbb{R}\right\} \approx \mathrm{U}(1),
$$

and

$$
I_{\omega_{1}}\left(0^{+}(M)\right) \approx C_{\mathrm{SO}(2 m)}(\mathrm{U}(m)),
$$

where $\mathrm{U}(m)$ is taken as a subgroup of $\mathrm{SO}(2 m)$ in the real representation.

\subsubsection{Lemma.}

$$
C_{\mathrm{SO}(2 m)}(\mathrm{U}(m))=Z(\mathrm{U}(m)) \approx \mathrm{U}(1) .
$$

Proof. In the real representation,

$$
J=\left(\begin{array}{cc}
0 & I_{m} \\
-I_{m} & 0
\end{array}\right) \in \mathrm{U}(m),
$$

where $I_{m}$ is the $m \times m$ real identity matrix. Let $A \in C_{\mathrm{SO}(2 m)}(\mathrm{U}(m))$. Since $A$ commutes with $J, A \in G L(m, \mathbb{C})$. Thus $A \in G L(m, \mathbb{C}) \cap \mathrm{SO}(2 m)=\mathrm{U}(m)$, and so $A \in \mathrm{U}(m)$. Since $Z(\mathrm{U}(m)) \subseteq C_{\mathrm{SO}(2 m)}(\mathrm{U}(m))$, we have equality.

Thus in the above example, the internal symmetry groups of $\omega_{0}$ and $\omega_{1}$ are isomorphic,

$$
I_{\omega_{1}}\left(0^{+}(M)\right) \approx I_{\omega_{0}}(U(M)) \approx Z(U(m)) \approx U(1) .
$$

3.7. Example. Let $G=\mathrm{SU}(n), n \geqq 2$, and let $P(M, G)=M \times \mathrm{SU}(n)$ be the product bundle, $\operatorname{dim} M \geqq 2$. Let $H$ be a closed connected proper subgroup of SU( $n)$ such that $H$ acts irreducibly on $\mathbb{C}^{n}$ [for example, in the real representation, $\mathrm{SO}(n) \subseteq \mathrm{SU}(n), n \geqq 2$, acts irreducibly on $\left.\mathbb{C}^{n}\right]$. Let $i: M \times H \rightarrow M \times \mathrm{SU}(n)$ denote the reduction of SU(n) to $H$. Let $\omega_{0}$ be a connection on $M \times H$ such that $\operatorname{Hol}\left(\omega_{0}\right)=H$, and let $\omega_{1}$ be the connection pushed over to $M \times \operatorname{SU}(n)$. Then $\operatorname{Hol}\left(\omega_{1}\right)$ $=\operatorname{Hol}\left(\omega_{0}\right)=H$, and so

$$
I_{\omega_{1}}(M \times \mathrm{SU}(n)) \approx C_{\mathrm{SU}(n)}(H)=Z(\mathrm{SU}(n))=\left\{e^{\frac{2 \pi i k}{n}} I_{n} \mid k=0,1, \ldots, n-1\right\},
$$


since by Schur's Lemma (valid for any field), if $H$ acts irreducibly on $\mathbb{C}^{n}$, then $C_{\mathrm{SU}(n)}(H)=Z(\mathrm{SU}(n)) . \omega_{1}$ is an example of a generic reducible connection (see Sect. 5).

3.8. Example. Let $P(M, G)$ be a PFB such that the total space $P$ is connected, and such that $G$ is a discrete Lie group. Then $\mathscr{5}=\{0\}$, and $\pi: P \rightarrow M$ is a regular covering manifold of $M$. Let

$$
\operatorname{Cov}(P)=\{F \in \operatorname{Diff}(P) \mid \pi \circ F=F\}
$$

denote the covering transformations of $P$. Then

$$
\operatorname{Cov}(P) \approx G \approx \pi_{1}\left(M, x_{0}\right) / \pi_{*}\left(\pi_{1}\left(P, p_{0}\right)\right),
$$

where $\pi_{1}$ denotes the first homotopy group of $M$ (respectively $P$ ) based at $x_{0} \in M$ $\left[\right.$ respectively $\left.p_{0} \in \pi^{-1}\left(x_{0}\right)\right]$. Also, $\operatorname{Aut}(P) \subseteq \operatorname{Cov}(P)$, and so $\operatorname{Aut}(P) \approx Z(G)$.

Since $\mathfrak{G}=\{0\}$, there exists only one connection $\omega_{0}=0$ on $P$, which is flat. Moreover, since every curve in $P$ is horizontal, $\operatorname{Hol}\left(\omega_{0}\right)=G$. Thus

$$
I_{\omega_{0}}(P) \approx C_{G}(\operatorname{Hol}(\omega)) \approx Z(G),
$$

so that $I_{\omega_{0}}(P) \approx Z(G)$. Note that since $\omega_{0}=0, I_{\omega_{0}}(P)$ is the entire group $\operatorname{Aut}(P)$, which is, however, only the 0-dimensional Lie group $Z(G)$. Also, see Proposition 5.4.

Finally, we give an example where the holonomy group is not a closed subgroup of the structure group $G$.

3.9. Example. Let $P\left(S^{1}, S^{1}\right)=S^{1} \times S^{1}$ be the product bundle, where $S^{1}=\mathrm{U}(1)=\left\{e^{i \omega} \mid \omega \in \mathbb{R}\right\}$. Identify $S^{1} \times S^{1}$ with the square $E$ of length $2 \pi$ in $\mathbb{R}^{2}$ with opposite edges identified. Let $\lambda$ be a fixed irrational real number, and for

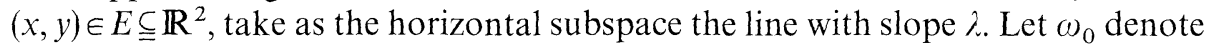
the corresponding connection 1 -form. Then since any connection over a 1-dimensional manifold is flat, $\omega_{0}$ is flat, and its holonomy group is the discrete subgroup of $\mathrm{U}(1)$,

$$
\operatorname{Hol}\left(\omega_{0}\right)=\left\{e^{i n \lambda} \mid n \in \mathbb{Z}\right\} \approx Z .
$$

Thus Hol $\left(\omega_{0}\right)$ is not a closed Lie subgroup of U(1). Since the structure group is Abelian, by Example 3.1, $I_{\omega_{0}}(P)=\mathrm{U}(1)$.

If $\lambda$ is a fixed rational number, and if the horizontal subspaces are taken as the lines with slope $\lambda$, then the holonomy sub-bundles are the $n$-fold covering manifolds $S^{1} \rightarrow S^{1}, z \rightarrow z^{n}$, for some $n \in Z^{+}$depending on $\lambda$, and the holonomy group is

$$
\operatorname{Hol}(\omega)=\left\{e^{\frac{2 \pi i k}{n}} \mid k=0,1, \ldots, n-1\right\} \approx Z_{n}
$$

the cyclic group of order $n$.

Thus the possible holonomy groups for connections on $S^{1} \times S^{1}$ are $Z$ and $Z_{n}$, $n \in Z^{+}$, and each of these holonomy groups is attained. 
Remark. In contrast, on the "cylindrical" PFB $P\left(S^{1}, \mathbb{R}\right)=S^{1} \times \mathbb{R}$, the holonomy sub-bundles of each connection produces either a spiral or circular foliation of $S^{1} \times \mathbb{R}$. Thus the possible holonomy groups are integer multiples of the vertical distance $d \geqq 0$ attained in one spiral. Thus the possible holonomy groups are

$$
Z d=\{n d \mid n \in Z, d \geqq 0\} \approx\left\{\begin{array}{lll}
\{0\} & \text { if } & d=0 \\
Z & \text { if } & d>0
\end{array} .\right.
$$

\section{Connections with Internal Symmetry Group as Large as Possible}

From the realization of $I_{\omega}(P)$ as a closed subgroup of $G$ by the map

$$
\operatorname{eval}_{p_{0}}: I_{\omega}(P), \rightarrow C_{G}\left(\operatorname{Hol}_{p_{0}}(\omega)\right) \cong G, \quad F \mapsto u_{F}\left(p_{0}\right),
$$

we see that $I_{\omega}(P)$ cannot exceed $G$. Thus we have the general estimate on the dimension of $I_{\omega}(P), \operatorname{dim} I_{\omega}(P) \leqq \operatorname{dim} G=k$.

It is of interest to know when $I_{\omega}(P)$ is as large as possible, i.e., when $I_{\omega}(P)$ is isomorphic to $G$. In this case we shall say that $I_{\omega}(P)$ (or that $\omega$ ) is maximal. If the weaker condition $\operatorname{dim} I_{\omega}(P)=k$ holds, we shall say that $I_{\omega}(P)$ has maximal dimension. Again, we note that $I_{\omega}(P) \approx G$ does not imply that $I_{\omega}(P)$ is equal to $\left\{R_{a} \mid a \in G\right\}$, but merely that the internal symmetry group is isomorphic to $G$ (see last paragraph of Sect. 2).

In Lagrangian field theories, there is some confusion over the role played by the symmetry group $I_{\omega}(P)$ and by the structure group $G$ of the $\operatorname{PFB} P(M, G)$. The symmetry group $I_{\omega}(P)$ generates global internal conservation laws, whereas in general the structure group $G$ does not. Thus it is of considerable interest to know when these groups are isomorphic. Thus, although the cases discussed below are special and "rare," they are many of the cases that have been considered in great detail classically, often resulting in the unfortunate confusion between the different roles played by $I_{\omega}(P)$ and $G$. (See Fischer [1985] for more details regarding these differing roles.)

The formula of Theorem 2.3 gives an easy criterion in terms of the holonomy group for when $I_{\omega}(P) \approx G$.

4.1. Proposition. Let $P(M, G)$ be a PFB, and let $\omega \in \mathscr{C}(P)$. Then $I_{\omega}(P) \approx G$ if and only if $\operatorname{Hol}(\omega) \subseteq Z(G)$.

Proof. If $\operatorname{Hol}(\omega) \subseteq Z(G)$, then $C_{G}\left(\operatorname{Hol}((\omega))=G\right.$. Thus $I_{\omega}(P) \approx C_{G}(\operatorname{Hol}(\omega))=G$.

Conversely, if $I_{\omega}(P) \approx G$, then $C_{G}(\operatorname{Hol}(\omega))=G$. Thus for all $a \in G, a \cdot b=b \cdot a$ for all $b \in \operatorname{Hol}(\omega)$. Thus if $b \in \operatorname{Hol}(\omega)$, then $b \in Z(G)$, and so $\operatorname{Hol}(\omega) \subseteq Z(G)$.

Examples 3.1, 3.2, and 3.3 are special cases of the above criterion. In Example 3.1, $G$ is Abelian and so $\operatorname{Hol}(\omega) \subseteq Z(G)=G$ for all connections $\omega \in \mathscr{C}(P)$. In Examples 3.2 and 3.3, $\operatorname{Hol}(\omega)=\{e\} \cong Z(G)$ for all Lie groups $G$, and so again the above criterion is satisfied. Using Example 3.3 and Proposition 4.1, we can also give the following criterion for maximality of flat connections.

4.2. Proposition. Let $\omega$ be a flat connection on the PFB $P(M, G)$.

(i) If $M$ is simply connected, then $I_{\omega}(G) \approx G$.

(ii) If $G$ is connected and $\operatorname{Hol}(\omega)$ is a normal subgroup of $G$, then $I_{\omega}(P) \approx G$. 
Proof. (i) If $\omega$ is flat and $M$ is simply connected, then $\operatorname{Hol}(\omega)=\{e\}$, and so $I_{\omega}(P) \approx G$ (see Example 3.3).

(ii) If $\omega$ is flat, $\operatorname{Hol}(\omega)$ is discrete. But a discrete normal subgroup of a connected topological group is central (see e.g., Greenberg-Harper [1981], p. 18), and so $\operatorname{Hol}(\omega) \subseteq Z(G)$. Thus by Proposition 4.1, $I_{\omega}(P) \approx G$.

We can also give a criterion for when $I_{\omega}(P)$ has maximal dimension. Let $G^{0}$ denote the connected component of the identity of $G$.

4.3. Proposition. Let $P(M, G)$ be a $\mathrm{PFB}$, let $k=\operatorname{dim} G$, and let $\omega \in \mathscr{C}(P)$. Then $\operatorname{dim} I_{\omega}(P)=k$ if and only if $\operatorname{Hol}(\omega) \subseteq C_{G}\left(G^{0}\right)$. In particular, if $G$ is connected, then $I_{\omega}(P) \approx G\left(I_{\omega}(P)\right.$ is maximal $)$ if and only if $\operatorname{dim} I_{\omega}(P)=k\left(I_{\omega}(P)\right.$ has maximal dimension).

Proof. From Theorem 2.3 and Lemma 1.5, $I_{\omega}(P) \approx C_{G}(\operatorname{Hol}(\omega)) \subseteq G$, and $C_{G}(\operatorname{Hol}(\omega))$ is a closed subgroup and hence a closed submanifold of $G$. Thus if $\operatorname{dim} I_{\omega}(P)=k$ $=\operatorname{dim} G$, then $C_{G}(\operatorname{Hol}(\omega)) \supseteqq G^{0}$. Thus if $a \in G^{0}, a \cdot b=b \cdot a$ for all $b \in \operatorname{Hol}(\omega)$, so if $b \in \operatorname{Hol}(\omega)$, then $b$ commutes with all $a \in G^{0}$, and so $b \in C_{G}\left(G^{0}\right)$. Thus $\operatorname{Hol}(\omega) \subseteq C_{G}\left(G^{0}\right)$.

Conversely, if $\operatorname{Hol}(\omega) \subseteq C_{G}\left(G^{0}\right)$, then if $b \in \operatorname{Hol}(\omega)$, then $b$ commutes with all elements in $G^{0}, b \cdot a=a \cdot b$ for all $a \in G^{0}$. Thus if $a \in G^{0}, a$ commutes with all $b \in \operatorname{Hol}(\omega)$. Thus

$$
G^{0} \cong C_{G}(\operatorname{Hol}(\omega)) \subseteq G
$$

and so $\operatorname{dim} C_{G}(\operatorname{Hol}(\omega))=k$.

4.4. Example. For an example of a connection with maximal dimension but which is not maximal, let $P=S^{1} \times O(2)$ and let $\omega_{0}$ be a connection on $P$ such that the horizontal subspaces on each component of $P$ have a fixed irrational slope $\lambda$ (see Example 3.9). Then $\operatorname{Hol}\left(\omega_{0}\right)=\left\{e^{i n \lambda} \mid n \in Z\right\}$, and $\overline{\operatorname{Hol}\left(\omega_{0}\right)}=\operatorname{SO}(2)$. Thus

$$
I_{\omega_{0}}(P) \approx C_{O(2)}\left(\operatorname{Hol}\left(\omega_{0}\right)\right)=C_{O(2)}\left(\overline{\left.\operatorname{Hol}\left(\omega_{0}\right)\right)}=C_{O(2)}(\operatorname{SO}(2))=\mathrm{SO}(2) .\right.
$$

Thus $\operatorname{dim} I_{\omega_{0}}(P)=1$, but $I_{\omega_{0}}(P) \neq O(2)$, so $\omega_{0}$ has maximal dimension but is not maximal.

In Example 3.3 we saw that if $M$ is simply connected and $\omega$ is flat, then $I_{\omega}(P) \approx G$. As a partial converse, we have the following.

4.5. Proposition. Let $P(M, G)$ be a PFB with $G$ semi-simple, and let $\omega \in \mathscr{C}(P)$.

(1) If $I_{o}(P) \approx G$, then $\omega$ is flat.

(2) If $G$ is connected and $\operatorname{dim} I_{\omega}(P)=k=\operatorname{dim} G$, then $\omega$ is flat.

Proof. If $I_{\omega}(P) \approx G$, then by Proposition 4.1, $\operatorname{Hol}(\omega) \subseteq Z(G)$. Since $G$ is semi-simple, its center $Z(G)$ is a discrete subgroup of $G$. Thus $\operatorname{Hol}(\omega)$ is a discrete subgroup of $G$, and so by the Ambrose-Singer Theorem (see Kobayashi-Nomizu [1963], p. 89), the connection is flat.

If $G$ is connected and $\operatorname{dim} I_{\omega}(P)=k$, then by Proposition $4.3, I_{\omega}(P) \approx G$, and so by (1) the connection is flat.

In Yang-Mills field theories, $G$ is often assumed to be compact, connected, and semi-simple (see e.g., Mitter [1980] or Singer [1978]). With these assumptions (even without $G$ being compact), a maximal dimension connection must be flat, thereby indicating the "scarcity" of maximal dimension connections. 


\section{Connections with Internal Symmetry Group as Small as Possible and Irreducible Connections}

We have seen that for all $a \in Z(G), R_{a}^{*} \omega=\omega$ for any connection $\omega \in \mathscr{C}(P)$. Thus the inclusion $Z(G) \subseteq I_{\omega}(P)$ holds for all connections $\omega$, so that $Z(G)$ is the smallest possible symmetry group for any connection. We shall say that the connection $\omega$ is generic if $I_{\omega}(P)=Z(G)$. Thus if $\omega$ is generic, $I_{\omega}(P)$ is as small as possible. By Theorem 2.3, a criterion for $\omega$ to be generic is given in terms of its holonomy group as follows:

$\omega$ is generic if and only if $C_{G}(\operatorname{Hol}(\omega))=Z(G)$.

We let

$$
\mathscr{C}_{0}(P)=\left\{\omega \in \mathscr{C}(P) \mid I_{\omega}(P)=Z(P)\right\}
$$

denote the space of generic connections on $P$. The term generic is justified by the fact that if $\mathscr{C}_{0}(P) \neq \phi$, then $\mathscr{C}_{0}(P)$ is an open dense subset of $\mathscr{C}(P)$ under the assumption that $P$ is a compact connected manifold [see Singer [1978] for this result for irreducible connections (see below)].

In the special case when $G$ is Abelian, $C_{G}(\operatorname{Hol}(\omega))=G=Z(G)$ for any connection $\omega$ (see Example 3.1), so that $\mathscr{C}_{0}(P)=\mathscr{C}(P)$. Thus in the Abelian case, every connection is both generic $\left(I_{\omega}(P)=Z(G)\right)$ and maximal $\left(I_{\omega}(P)=G\right)$, since $Z(G)=G$.

Returning to the general case, we remark that although $I_{\omega}(P)=Z(G)$ is constant as a function of $\omega \in \mathscr{C}_{0}(P)$, the holonomy group $\operatorname{Hol}(\omega)$ may depend on $\omega \in \mathscr{C}_{0}(P)$. For example, if $P=S^{1} \times S^{1}$ (see Example 3.9), then $\mathscr{C}_{0}(P)=\mathscr{C}(P)$, but $\operatorname{Hol}(\omega)$ depends on $\omega \in \mathscr{C}(P)$.

We shall also need the following result regarding generic connections.

5.1. Proposition. Let $P(M, G)$ be a PFB, and assume $\mathscr{C}_{0}(P) \neq \phi$. Let $\omega \in \mathscr{C}_{0}(P)$. If for some $p \in P, \operatorname{Hol}_{p}(\omega)$ is Abelian, then $G$ is Abelian.

Proof. Let $H=\operatorname{Hol}_{p}(\omega)$ be Abelian. If $a \in H, a \cdot b=b \cdot a$ for all $b \in H$, and so $a \in C_{G}(H)$. Thus $H \subseteq C_{G}(H)$. Since $\omega \in \mathscr{C}_{0}(P) \neq \phi, C_{G}(H)=Z(G)$. Thus $H \subseteq Z(G)$, and so $C_{G}(H)=G$. Thus $Z(G)=G$, and so $G$ is Abelian.

Remark. Note that under the conditions of the proposition, $\omega$ is also maximal.

We now go on to study the irreducible connections, which turn out to be a large sub-class of the space of generic connections. Unfortunately, there are at least three definitions of an irreducible connection in the literature.

If $\omega$ is a connection on $P(M, G)$, then by the Holonomy Reduction Theorem (see Kobayashi-Nomizu [1963], p. 83), $\omega$ can be reduced to a connection on the holonomy sub-bundle $P\left(p_{0}\right)=Q\left(M, \mathrm{Hol}_{p_{0}}(\omega)\right)$. Thus it is reasonable to define a connection to be irreducible if $\operatorname{Hol}(\omega)=G$, and this is in fact done by several authors (see e.g. Atiyah-Jones [1978], p. 101, Daniel-Viallet [1980], p. 186, or Rawnsley [1978], p. 35). However, if $\operatorname{Hol}_{p_{0}}(\omega)$ is not a closed subgroup of $G, P\left(p_{0}\right)$ will only be an immersed and not an embedded submanifold of $P$. The connection $\omega$ could then be reduced to a connection on the closed sub-bundle $\overline{P\left(p_{0}\right)}$ $=Q\left(M, \overline{\left.\operatorname{Hol}_{p_{0}}(\omega)\right)}\right.$. Thus if one wishes to rule out reductions of $\omega$ to immersed subbundles as not being "proper" reductions, then an irreducible connection should 
be defined as a connection such that $\overline{\operatorname{Hol}(\omega)}=G$. This weaker definition is chosen by other authors (see e.g. Atiyah, Hitchin, and Singer [1978], p. 442). Finally, for a matrix Lie group $G \subseteq G L(n, \mathbb{R})$, Singer [1978], p. 9, defines a connection to be irreducible if its holonomy group $\operatorname{Hol}(\omega) \subseteq G$ acts irreducibly on $\mathbb{R}^{n}$.

Since for a general PFB this last definition requires a representation of the structure group $G$ on $\mathbb{R}^{n}$, we do not take it over to define an irreducible connection on $P(M, G)$. Since non-closed holonomy groups are a fact of life in the theory of connections, we will consider reductions to immersed sub-bundles as being bona fide reductions. Thus we end up with the following.

5.2. Definition. Let $\omega \in \mathscr{C}(P)$ be a connection on $P(M, G)$. Then $\omega$ is irreducible if $\operatorname{Hol}(\omega)=G$, and $\omega$ is weakly irreducible if $\operatorname{Hol}(\omega)=G$. $\omega$ is irreducible if $\operatorname{Hol}(\omega) \neq G$, and $\omega$ is weakly reducible if $\operatorname{Hol}(\omega) \neq G$.

Let

$$
\mathscr{C}_{I}(P)=\{\omega \in \mathscr{C}(P) \mid \operatorname{Hol}(\omega)=G\}
$$

denote the space of irreducible connections on $P$, and let

$$
\left.\mathscr{C}_{W}(P)=\{\omega) \in \mathscr{C}(P) \mid \overline{\operatorname{Hol}(\omega)}=G\right\}
$$

denote the space of weakly irreducible connections on $P$. Thus

$$
\mathscr{C}_{I}(P) \cong \mathscr{C}_{W}(P) \cong \mathscr{C}(P) .
$$

5.3. Proposition. If $\omega$ is a weakly irreducible connection on $P(M, G)$, then

$$
I_{\omega}(P)=Z(G)=\left\{R_{a}: P \rightarrow P \mid a \in Z(G)\right\} .
$$

Thus every weakly irreducible connection is generic, and in particular, every irreducible connection is generic.

Proof. Since $\omega$ is weakly irreducible, $\overline{\operatorname{Hol}(\omega)}=G$. Thus from Theorem 2.3 and Lemma 1.5,

$$
I_{\omega}(P) \approx C_{G}(\operatorname{Hol}(\omega))=C_{G} \overline{(\operatorname{Hol}(\omega))}=C_{G}(G)=Z(G) .
$$

Since for any connection, $Z(G) \subseteq I_{\omega}(P)$, it follows that $I_{\omega}(P)=Z(G)$.

From the above proposition, we now have the following inclusions:

$$
\mathscr{C}_{I}(P) \subseteq \mathscr{C}_{W}(P) \subseteq \mathscr{C}_{0}(P) \subseteq \mathscr{C}(P) .
$$

In general, the inclusion $\mathscr{C}_{W}(P) \subseteq \mathscr{C}_{0}(P)$ is proper. For example, if $G$ is Abelian, $\mathscr{C}_{0}(P)=\mathscr{C}(P)$ (see Example 3.1), and so any weakly reducible connection $(\overline{\operatorname{Hol}(\omega)} \neq G)$ will be in $\mathscr{C}_{0}(P)$ but not in $\mathscr{C}_{W}(P)$. Another less trivial example is the canonical connection $\omega_{0}$ on the frame bundle $L\left(S^{n}\right)$ with $n \geqq 3$ (see Example 3.4). Here $\operatorname{Hol}\left(\omega_{0}\right)=\operatorname{SO}(n)$, and so $\omega_{0}$ is reducible and so weakly reducible to the proper closed sub-bundle $0^{+}\left(S^{n}\right)$, but $I_{\omega_{0}}\left(L\left(S^{n}\right)\right)=Z(G)$, and so $\omega_{0} \in \mathscr{C}_{0}\left(L\left(S^{n}\right)\right)$ is generic, but $\omega_{0} \notin \mathscr{C}_{W}(P)$.

The above inclusion is proper even in the case when $G$ is compact, connected, and semi-simple, as shown in Example 3.7, where a generic connection $\omega_{1}$ is given on $M \times \mathrm{SU}(n), \operatorname{dim} M \geqq 2, n \geqq 2$. By construction, $\omega_{1}$ is reducible and so strongly reducible to a connection $\omega_{0}$ on the proper closed sub-bundle, $M \times H$.

Regarding the existence of irreducible connections, we have the following. 
5.4. Proposition. Let $P(M, G)$ be a PFB where the total space $P$ is connected and $\operatorname{dim} M \geqq 2$. Then there exists an irreducible connection on $P$, so that $\mathscr{C}_{I}(P) \neq \phi$, $\mathscr{C}_{W}(P) \neq \phi$, and $\mathscr{C}_{0}(P) \neq \phi$.

Proof. By a theorem of Kobayashi-Nomizu [1963], p. 90, there exists a connection $\omega_{0}$ such that $\operatorname{Hol}\left(\omega_{0}\right)=G$. This $\omega_{0}$ is irreducible, and so $\omega_{0} \in \mathscr{C}_{I}(P) \cong \mathscr{C}_{W}(P) \cong \mathscr{C}_{0}(P)$.

The one-dimensional case is an interesting curiosity. The variety of possibilities, listed below, is somewhat unexpected.

5.5. Proposition. Let $\operatorname{dim} M=1$, so that $M$ is either diffeomorphic to $\mathbb{R}$ or to $S^{1}$, and let $P(M, G)$ be a PFB over $M$.

If $M \approx \mathbb{R}$, then $P(M, G) \approx \mathbb{R} \times G$. If $G$ is Abelian, then $\mathscr{C}_{0}(P)=\mathscr{C}(P) \neq \phi$. If $G$ is not Abelian, then $\mathscr{C}_{I}(P)=\mathscr{C}_{W}(P)=\mathscr{C}_{0}(P)=\phi$. If $G \neq\{e\}$ ( $G$ Abelian or not $)$, then $\mathscr{C}_{I}(P)=\mathscr{C}_{W}(P)=\phi$. If $G=\{e\}$, then $\left.\mathscr{C}_{I}(P)=\mathscr{C}_{W}(P)=\mathscr{C}_{0}(P)=\mathscr{C}(P)=\{\omega)_{0}\right\} \neq \phi$, where $\omega_{0}=0$ is the zero connection on $P(\mathbb{R}\{e\})$ ( see Example 3.8).

If $M \approx S^{1}$, and if $G$ is Abelian, then $\mathscr{C}_{0}(P)=\mathscr{C}(P) \neq \phi$. If $G$ is not Abelian then $\mathscr{C}_{I}(P)=\mathscr{C}_{W}(P)=\mathscr{C}_{0}(P)=\phi$. If $P\left(S^{1}, G\right)$ is connected and $\operatorname{dim} G=0$, then $G$ is isomorphic to either $Z$ or $Z_{n}$ for some $n \in Z^{+}$, and $P\left(S^{1}, G\right)$ is bundle isomorphic to either covering manifold $\mathbb{R} \rightarrow S^{1} ; \lambda \mapsto e^{i \lambda}$, for $G \approx Z$, or $S^{1} \rightarrow S^{1} ; z \mapsto z^{n}$ for $G \approx Z_{n}$, and where $S^{1}=\{z \in \mathbb{C} \mid z \bar{z}=1\}$. In either case

$$
\mathscr{C}_{W}(P)=\mathscr{C}_{I}(P)=\mathscr{C}_{0}(P)=\mathscr{C}(P)=\left\{\omega_{0}\right\} \neq \phi,
$$

where $\omega_{0}=0$.

If $G$ is Abelian and $\operatorname{dim} G \geqq 1$, then $\mathscr{C}_{I}(P)=\phi$, but whether $\mathscr{C}_{W}(P)$ is empty or not depends on the bundle $P\left(S^{1}, G\right)$.

Proof. From Example 3.1 and Sect. 5, if $G$ is Abelian, then $\mathscr{C}_{0}(P)=\mathscr{C}(P) \neq \phi$, for any PFB $P(M, G)$.

If $\operatorname{dim} M=1$, then the curvature 2 -form of connection $\omega$ on $P(M, G)$ must vanish, so that any connection on $P(M, G)$ must be flat.

Now let $M \approx \mathbb{R}$. Since $\mathbb{R}$ is contractable, $P(\mathbb{R}, G)$ is bundle isomorphic to $\mathbb{R} \times G$. Let $\omega \in \mathscr{C}(P)$. Since $\omega$ is flat and $\mathbb{R}$ is simply connected, Hol $(\omega)=\{e\}$. Now suppose $\mathscr{C}_{0}(P) \neq \phi$. Let $\omega_{0} \in \mathscr{C}_{0}(P)$, so that $Z(G)=C_{G}\left(\operatorname{Hol}\left(\omega_{0}\right)\right)=C_{G}(\{e\})=G$, and so $G$ is Abelian. Thus if $G$ is not Abelian, $\mathscr{C}_{0}(P)=\phi$, and so $\mathscr{C}_{W}(P)=\mathscr{C}_{I}(P)$ $=\mathscr{C}_{0}(P)=\phi$.

Since $\operatorname{Hol}(\omega)=\{e\}$ for every connection $\omega \in \mathscr{C}(P)$, every connection is reducible to the zero connection $\omega_{0}=0$ on the closed holonomy sub-bundle $P\left(p_{0}\right)$ $=Q(M,\{e\})$. Thus if $G \neq\{e\}$, then $\mathscr{C}_{W}(P) \neq \phi$, and so also $\mathscr{C}_{I}(P)=\phi$. If $G=\{e\}$, then

$$
\mathscr{C}_{W}(P)=\mathscr{C}_{I}(P)=\mathscr{C}_{0}(P)=\mathscr{C}(P)=\left\{\omega_{0}\right\} \neq \phi .
$$

Now let $M \approx S^{1}$. For $p_{0} \in P\left(S^{1}, G\right)$, let $x_{0}=\pi\left(p_{0}\right) \in S^{1}$. For $\omega \in \mathscr{C}(P)$, le

$$
h: \pi_{1}\left(S^{1}, x_{0}\right) \rightarrow \operatorname{Hol}_{p_{0}}(\omega)
$$

denote the holonomy homomorphism (see Kobayashi-Nomizu [1963], p. 93). Since $\pi_{1}\left(S^{1}, x_{0}\right) \approx Z$ and since $h$ is surjective, $\operatorname{Hol}_{p_{0}}(\omega) \approx \frac{Z}{\operatorname{ker} h}$ is isomorphic to 
either $Z$ or $Z_{n}=\frac{Z}{n Z}$ for some $n \in Z^{+}$. Thus $\operatorname{Hol}_{p_{0}}(\omega)$ is Abelian, and so by Proposition 5.1, if $\mathscr{C}_{0}(P) \neq \phi$, then $G$ is Abelian. Thus if $G$ is not Abelian, $\mathscr{C}_{0}(P)=\phi$, and so $\mathscr{C}_{W}(P)=\mathscr{C}_{I}(P)=\mathscr{C}_{0}(P)=\phi$.

If $\operatorname{dim} G=0, G$ is a discrete Lie group, and so if $P$ is connected, by Example 3.8, $P \rightarrow S^{1}$ is a regular covering manifold of $S^{1}$. But every connected covering manifold of $S^{1}$ is equivalent to either $\mathbb{R} \rightarrow S^{1}, \lambda \rightarrow e^{i \lambda}$, or $S^{1} \rightarrow S^{1}, z \rightarrow z^{n}$, for some $n \in Z^{+}$(see Spanier [1966], p. 80). Thus $G$ must be either $Z$ or $Z_{n}$ for some $n \in Z^{+}$. In either case, there is only one connection $\omega_{0}=0$ on $P$, and $\operatorname{Hol}\left(\omega_{0}\right)=G$ (see Example 3.8). Thus

$$
\mathscr{C}_{I}(P)=\mathscr{C}_{W}(P)=\mathscr{C}_{0}(P)=\mathscr{C}(P)=\left\{\omega_{0}\right\} \neq \phi .
$$

Now assume $\operatorname{dim} G \geqq 1, G$ Abelian. Since every connection $\omega$ on $P\left(S^{1}, G\right)$ is flat, the holonomy groups must be discrete subgroups of $G$. Thus if $\operatorname{dim} G \geqq 1$, $\operatorname{Hol}(\omega) \neq G$ for any connection $\omega$ on $P$. Hence $\mathscr{C}_{I}(P)=\phi$.

Example 3.9 with $P=S^{1} \times S^{1}$ and with connection $\omega_{0}$ determined by horizontal subspaces with irrational slope gives $\overline{\operatorname{Hol}\left(\omega_{0}\right)}=S^{1}$, and hence $\omega_{0} \in \mathscr{C}_{W}(P) \neq \phi$. On the other hand, the holonomy group of any connection on $P=S^{1} \times \mathbb{R}$ is a discrete subgroup of $\mathbb{R}$ (see Example 3.9), and so $\overline{\operatorname{Hol}(\omega)} \neq G$ for any connection $\omega$ on $P$. Hence $\mathscr{C}_{W}(P)=\phi$. Thus whether $\mathscr{C}_{W}(P)$ is empty or not depends on the bundle $P\left(S^{1}, G\right)$.

We summarize the above results in the following tables, with $\omega_{0}=0$.

Subspaces of $\mathscr{C}(P)$

\begin{tabular}{llll}
\hline Group $G$ & $\mathscr{C}_{I}(P)$ & $\mathscr{C}_{W}(P)$ & $\mathscr{C}_{0}(P)$ \\
\hline $\mathrm{G}=\{e\}$ & $\left\{\omega_{0}\right\}$ & $\left\{\omega_{0}\right\}$ & $\left\{\omega_{0}\right\}$ \\
\hline$G$ Abelian, $G \neq\{e\}$ & $\phi$ & $\phi$ & $\mathscr{C}_{0}(P)=\mathscr{C}(P) \neq \phi$ \\
\hline$G$ not Abelian & $\phi$ & $\phi$ & $\phi$ \\
\hline
\end{tabular}

Case $1, M \approx \mathbb{R}$

Subspaces of $\mathscr{C}(P)$

\begin{tabular}{llll}
\hline Group $G$ & $\mathscr{C}_{I}(P)$ & $\mathscr{C}_{W}(P)$ & $\mathscr{C}_{0}(P)$ \\
\hline $\begin{array}{l}P\left(S^{1}, G\right) \text { connected, } \\
\operatorname{dim} G=0 \Rightarrow G \approx Z \text { or } Z_{n},\end{array}$ & $\left\{\begin{array}{l}\left\{\omega_{0}\right\} \\
n \in Z^{+}\end{array}\right.$ & $\begin{array}{l}\left\{\omega_{0}\right\} \\
\left\{\omega_{0}\right\}\end{array}$ & $\begin{array}{l}\left\{\omega_{0}\right\} \\
\left\{\omega_{0}\right\}\end{array}$ \\
\hline$G$ Abelian, $\operatorname{dim} G \geqq 1$ & $\phi$ & $\begin{array}{c}\text { depends on } \\
P(S, G)\end{array}$ & $\mathscr{C}_{0}(P)=\mathscr{C}(P) \neq \phi$ \\
\hline$G$ not Abelian & & $\phi$ & $\phi$ \\
\hline
\end{tabular}




\section{6. $I_{\omega}(P)$ as a Lie Group of Transformations}

In Sect. 2 we showed that $I_{\omega}(P, G)$, and hence $I_{\omega}(P)$ has a Lie group structure. In this section we show that $I_{\omega}(P)$ is a Lie transformation group that acts properly and freely on $P$.

Let $P(M, G)$ be a PFB over $M$, let $g$ be a Riemannian metric on $M(M$ is paracompact and hence admits Riemannian metrics), let $\omega \in \mathscr{C}(P)$, and let $\gamma$ be a positive-definite inner product on $\mathbf{6}$. We are not assuming that $\gamma$ is adjointinvariant. Indeed, such a $\gamma$ does not exist on a general Lie algebra. Using $g, \omega$, and $\gamma$, we construct a Riemannina metric $g$ on $P$ by $\hat{g}=\pi^{*} g+\gamma \cdot(\omega \otimes \omega)$, so that

$$
g(p) \cdot\left(Z_{1}, Z_{2}\right)=g(x)\left(X_{1}, X_{2}\right)+\gamma \cdot\left(\omega\left(Z_{1}\right), \omega\left(Z_{2}\right)\right),
$$

where $p \in P, Z_{1}, Z_{2} \in T_{p} P, x=\pi(p)$, and $X_{1}=T_{p} \pi \cdot Z_{1}, X_{2}=T_{p} \pi \cdot Z_{2} \in T_{x} M$. Note that if $\gamma$ is not Ad-invariant, then $g$ is not $G$-invariant $\left(R_{a}^{*} \hat{g} \neq \hat{g}\right.$ for all $\left.a \in G\right)$. However, we shall see below that $g$ is $I_{\omega}(P)$-invariant. This observation is the key to Theorem 6.2 below.

Let

$$
I_{\hat{g}}(P)=\left\{H \in \operatorname{Diff}(P) \mid H^{*} \hat{g}=\hat{g}\right\}
$$

denote the group of isometries of $P$. Note that an isometry need not be an automorphism of $P$. A classical result of Myers and Steenrod ([1939]) states that if $P$ is connected, then with respect to the compact open topology, $I_{\hat{g}}(P)$ is a Lie transformation group of $P$; i.e., $I_{\hat{g}}(P)$ has a Lie group structure such that the action $I_{\hat{g}}(P) \times P \rightarrow P,(H, p) \mapsto H(p)$ is a $C^{\infty}$ map. Also, the Lie algebra of $I_{\hat{g}}(P)$ consists of the complete Killing vector fields on $P$,

$$
\mathscr{I}_{\hat{g}}(P)=\left\{W \in \mathfrak{X}(P) \mid W \text { is complete and } L_{W} g=0\right\} .
$$

As part of this classical result, it is shown that if $\left\{H_{n}\right\}$ is a sequence in $I_{\hat{g}}(P)$ such that $H_{n}\left(p_{0}\right) \rightarrow p_{1}$, then there exists an $H \in I_{\hat{g}}(P)$ [with $\left.H\left(p_{0}\right)=p_{1}\right]$ and a subsequence $\left\{H_{n_{k}}\right\}$ of $\left\{H_{n}\right\}$ that converges to $H$ in the compact open topology (see Helgason [1962], p. 167 or Kobayashi-Nomizu [1963], p. 47). This latter result can be rephrased as follows.

6.1. Proposition. If $P$ is connected, then the action $I_{\hat{g}}(P) \times P \rightarrow P,(H, p) \mapsto H(p)$ is a proper action.

Proof. The above action is proper if the map $I_{\hat{g}}(P) \times P \rightarrow P \times P,(H, p) \mapsto(p, H(p))$ is a proper map, i.e., if the inverse image of a compact set is compact. This is equivalent to showing that if $\left\{p_{n}\right\}$ is a sequence that converges in $P$, and $\left\{H_{n}\right\}$ is a sequence in $I_{\hat{g}}(P)$ such that $\left\{H_{n}\left(p_{n}\right)\right\}$ converges in $P$, then $\left\{H_{n}\right\}$ has a convergent subsequence in $I_{\hat{g}}(P)$. Thus let $\left(p_{n}, H_{n}\left(p_{n}\right)\right) \rightarrow\left(p_{0}, p_{1}\right) \in P \times P$. Let $d$ be the metric on $P$ associated with $\hat{\mathrm{g}}$. Then since $\hat{\mathrm{g}}$ is $I_{\hat{g}}(P)$-invariant, so is $d$. Thus

$$
d\left(H_{n}\left(p_{0}\right), p_{1}\right) \leqq d\left(H_{n}\left(p_{0}\right), H_{n}\left(p_{n}\right)\right)+d\left(H_{n}\left(p_{n}\right), p_{1}\right)=d\left(p_{0}, p_{n}\right)+d\left(H_{n}\left(p_{n}\right), p_{1}\right) .
$$

Since $p_{n} \rightarrow p_{0}$ and $H_{n}\left(p_{n}\right) \rightarrow p_{1}$, the right-hand side converges to zero. Hence $H_{n}\left(p_{0}\right) \rightarrow p_{1}$. Thus by the classical result, there exists a convergent subsequence $\left\{H_{n_{k}}\right\}$ in $I_{g}(P)$. Hence the action is proper.

Our main theorem is now the following. 
6.2. Theorem. Let $P(M, G)$ be a $\mathrm{PFB}$, let $\omega$ be a connection on $P(M, G)$, and let

$$
I_{\omega}(P)=\left\{F \in \operatorname{Aut}(P) \mid F^{*} \omega=\omega\right\}
$$

denote the internal symmetry group of $\omega$. Assume that the total space P is a connected manifold. Then with respect to the compact open topology on $I_{\omega}(P)$,

(1) $I_{\omega}(P)$ is a Lie transformation group of $P$, i.e., the action $\Psi: I_{\omega}(P) \times P \rightarrow P$, $(F, p) \mapsto F(p)$ is a $C^{\infty}$ action.

(2) The Lie algebra of $I_{\omega}(P)$ is:

$$
\mathscr{F}_{\omega}(P)=\left\{T \in \mathfrak{X}_{G}^{\mathrm{vert}}(P) \mid L_{T} \omega=D \varphi_{T}=0\right\} .
$$

(3) The action $\Psi$ is proper and free.

(4) $\operatorname{dim} I_{\omega}(P) \leqq \operatorname{dim}(\mathfrak{5}$.

(5) For $p \in P$, the orbits

$$
\mathcal{O}_{P}=\left\{F(p) \mid F \in I_{\omega}(P)\right\}
$$

are closed submanifolds of $P$, and the orbit maps $\Psi_{p}: I_{\omega}(P) \rightarrow \mathcal{O}_{P} \subseteq P, F \mapsto F(p)$ are diffeomorphisms onto the orbits. The differentiable structure on $I_{\omega}(P)$ is given by the embedding of $I_{\omega}(P)$ onto the closed submanifold $\mathcal{O}_{p}$.

(6) The orbit space

$$
I_{\omega}(P) \backslash P=\left\{\mathcal{O}_{p} \mid p \in P\right\}
$$

in the quotient topology has the structure of a $C^{\infty}$ manifold such that the orbit projection map $P \rightarrow I_{\omega}(P) \backslash P, p \mapsto \mathcal{O}_{p}$ is a submersion, and is also a left principal fiber bundle with total space $P$, base space $I_{\omega}(P) \backslash P$, and structure group $I_{\omega}(P)$.

(7) For $p \in P$, the map

$$
I_{\omega}(P) \rightarrow C_{G}\left(\operatorname{Hol}_{p}(\omega)\right), \quad F \mapsto u_{F}(p)
$$

is a Lie group isomorphism.

(8) If the structure group $G$ of the $\operatorname{PFB} P(M, G)$ is compact, then $I_{\omega}(P)$ is a compact Lie transformation group.

Proof. (1) For $F \in \operatorname{AUT}(P)$, let $f=\hat{\pi}(F) \in \operatorname{Diff}(M)$, so that $\pi \circ F=f \circ \pi$. Then

$$
F^{*} \hat{g}=F^{*}\left(\pi^{*} g\right)+F^{*}(\gamma \cdot(\omega \otimes \omega))=\pi^{*}\left(f^{*} g\right)+\gamma \cdot\left(F^{*} \omega \otimes F^{*} \omega\right),
$$

where $F^{*}$ pulls through the second term because of the bilinearity of $\gamma$. Thus if $F \in I_{\omega}(P), \hat{\pi}(F)=\mathrm{id}_{M}$, and $F^{*} \omega=\omega$, so that $F^{*} \hat{g}=\pi^{*} g+\gamma \cdot(\omega \otimes \omega)=\hat{g}$. Thus $F \in I_{\hat{g}}(P)$, and so $I_{\omega}(P) \subseteq I_{\hat{g}}(P)$.

Moreover, in the compact open topology, $F^{*} \omega=\omega$ is clearly a closed condition, and so $I_{\omega}(P)$ is a closed subgroup of $I_{\hat{g}}(P)$. From the classical result stated above, $I_{\hat{g}}(P)$ is a Lie transformation group. Since any closed subgroup of a Lie transformation group is also a Lie transformation group, it follows that $I_{\omega}(P)$ is a Lie transformation group.

(2) Let $F_{\lambda}$ be a one-parameter group of transformations in $I_{\omega}(P)$, and let

$$
T=\left.\frac{d F_{\lambda}}{d \lambda}\right|_{\lambda=0} \in \mathfrak{X}_{G}^{\mathrm{vert}}(P)
$$

be its generator. Then $F_{\lambda}^{*} \omega=\omega$, and so $L_{T} \omega=0$. Thus $T \in \mathscr{I}_{()}(P)$. 
Conversely, let $T \in \mathscr{I}_{\omega}(P) \subseteq \mathfrak{X}_{G}^{\mathrm{vert}}(P)$. Then since $G$-invariant vertical vector fields are automatically complete, $T$ is complete. Let $F_{\lambda} \in \operatorname{Aut}(P)$ be its flow. Since $L_{T} \omega=0, F_{\lambda}^{*} \omega=\omega\left(\right.$ see Kobayashi-Nomizu [1963], p. 33), and so $F_{\lambda} \in I_{\omega}(P)$.

(3) The action is proper since the action of $I_{\hat{g}}(P)$ on $P$ is proper (see Proposition 6.1), and since the restriction of a proper action to any closed subgroup is also a proper action.

Since $P$ is connected, $M$ is necessarily connected. Thus the action is free by Proposition 2.1 and Remark 2 following it. Note that the action of $I_{\hat{g}}(P)$ on $P$ is not necessarily free.

(4) Fix $p \in P$. Then the map $\mathscr{I}_{\omega}(P) \rightarrow(\mathfrak{5}, T \mapsto \omega(p) \cdot T(p)$ is an injection by Proposition 2.1 (and Remark 2 following it) and so $\operatorname{dim} I_{\omega}(P)=\operatorname{dim} \mathscr{I}_{\omega}(P) \leqq \operatorname{dim}(\mathfrak{6}$.

(5) and (6) follow for any smooth, proper and free action (see AbrahamMarsden [1978], pp. 266 and 276). That the differentiable structure on $I_{\omega}(P)$ is induced by the embedding $\Phi_{p}: I_{\omega}(P) \rightarrow \mathcal{C}_{p}, F \mapsto F(p)$, follows from the corresponding result for $I_{\hat{g}}(P)$ (see Kobayashi [1969], pp. 15 and 41).

(7) Since $C_{G}\left(\operatorname{Hol}_{p}(\omega)\right)$ is a closed subgroup of $G$, the map

$$
C_{G}\left(\operatorname{Hol}_{p}(\omega)\right) \rightarrow \mathcal{O}_{p} \subseteq P, \quad a \mapsto p \cdot a,
$$

is a diffeomorphism onto the orbit. Hence the Lie group structure on $I_{\omega}(P)$ induced by the embedding $I_{\omega}(P) \rightarrow \mathcal{O}_{p}, F \mapsto F(p)$, coincides with the Lie group structure on $I_{\omega}(P)$ induced by the group isomorphism

$$
I_{\omega}(P) \rightarrow C_{G}\left(\operatorname{Hol}_{p}(\omega)\right), \quad F \mapsto u_{F}(p) .
$$

Thus (7) is a restatement of Theorem 2.3, again using the fact that $M$ is connected. We also note again that all second countable Lie group structures on a Lie group are equivalent (see discussion following Proposition 2.3).

(8) Since $C_{g}\left(\Phi_{p}(\omega)\right)$ is a closed subgroup of $G$, if $G$ is compact, so is $C_{G}\left(\Phi_{p}(\omega)\right)$, and hence also $I_{\omega}(P)$.

Remarks. 1. A similar but modified formulation of the above theorem is available for the "action"

$$
I_{(0)}(P, G) \times P \rightarrow G, \quad(u, p) \mapsto u(p) .
$$

Thus $I_{\omega}(P, G)$ has a Lie group structure such that the above "action" is $C^{\infty}$, it is free in the sense that if $u(p)=e$, then $u \equiv e$, and it is proper in the sense that

$$
I_{\omega}(P, G) \times P \rightarrow P \times G, \quad(u, p) \mapsto(p, u(p))
$$

is a proper map.

2. As we have noted, if $P$ is connected, then $M$ is connected. However, $G$ need not be connected. On the other hand, if both $M$ and $G$ are connected, then $P$ is connected.

3. Note that if $G$ is compact, $\mathrm{Hol}(\omega)$ need not be compact (see Example 3.9). Thus it is of interest that $I_{\omega}(P) \approx C_{G}(\operatorname{Hol}(\omega))$ is compact [since $C_{G}(\mathrm{Hol}(\omega)$ is a closed subgroup of $G$ ]. Note the analogy with Riemannian geometry, where if $M$ is compact, then the isometry group $I_{g}(M)$ of a Riemannian metric $g$ is compact. Thus in this regard, compactness of the structure group $G$, causing $I_{(1)}(P)$ to be compact, is analogous to compactness of $M$, causing $I_{g}(M)$ to be compact. 
4. If $P$ has a finite number of components, then $I_{\hat{g}}(P)$ is still a Lie transformation group, but its action on $P$ is no longer proper. However, if $M$ is connected, the action of $I_{\omega}(P)$ on $P$ is still a proper action, inasmuch as $F \in I_{\omega}(P)$ is determined by its value at a point.

\section{The Lie Group of Transformations $I_{(g,(\omega)}(P)$}

As noted in the introduction, there are several finite dimensional subgroups of the infinite dimensional group $\operatorname{AUT}_{\omega}(P)$ which are Lie groups. If $P$ is a frame bundle, then we can consider the Lie group of affine transformations of $\omega$,

$$
A_{\omega}(M) \approx \operatorname{AUT}_{(\theta, \omega)}(P)=\operatorname{AUT}_{\theta}(P) \cap \operatorname{AUT}_{\omega}(P) \equiv I_{(\theta, \omega)}(P),
$$

where $\theta$ is now the canonical 1 -form on $P=L(M)$. For a general PFB, we considered in the previous section the Lie group $I_{\omega}(P)=\operatorname{Aut}(P) \cap \mathrm{AUT}_{\omega}(P)$. In this section we put a Riemannian metric $g$ on the base manifold $M$ and consider the larger subgroup

$$
I_{(q . \omega)}(P)=\left\{F \in \operatorname{AUT}(P) \mid F^{*} \omega=\omega \text { and } f^{*} g=g\right\} \supseteqq I_{\omega}(P),
$$

where $f=\hat{\pi}(F) \in \operatorname{Diff}(M)$ is the diffeomorphism of $M$ induced by the automorphism $F$ of $P$. Thus $I_{(g, \omega)}(P)$ is the group of automorphisms of $\omega$ that cover isometries of $g$.

Regarding $I_{(g, \omega)}(P)$ we have the following result.

7.1. Theorem. Let $P(M, G)$ be a PFB, let $\omega$ be a connection on $P$, and let $g$ be a Riemannian metric on $M$. Assume that the total space $P$ is a connected manifold. Then with respect to the compact open topology on $I_{(g, \omega)}(P)$,

(1) $I_{(g,(0)}(P)$ is a Lie transformation group of $P$, i.e., the action $\Psi$ :

$$
I_{(g . \omega)}(P) \times P \rightarrow P, \quad(F, p) \mapsto F(p)
$$

is a $C^{\infty}$ action.

(2) The Lie algebra of $I_{(g, \omega)}(P)$ is

$$
\begin{aligned}
\mathscr{I}_{(g \cdot())}(P)= & \left\{Z \in \mathfrak{X}_{G}(P) \mid Z \text { is a complete vector field on } P,\right. \\
& \text { and } \left.L_{Z} \omega=0 \text { and } L_{X} g=0 \text {, where } X=\pi_{*} Z \in \mathfrak{X}(M)\right\} .
\end{aligned}
$$

(3) The action $\psi$ is a proper action.

(4) $\operatorname{dim} I_{(g . \omega)}(P) \leqq \frac{1}{2} n(n+1)+k$, where $k=\operatorname{dim} G$.

(5) The isotropy group at an arbitrary point $p_{0} \in P$,

$$
\left(I_{(g, \omega))}(P)\right)_{p_{0}}=\left\{F \in I_{(g, \omega)}(P) \mid F\left(p_{0}\right)=p_{0}\right\} .
$$

is compact.

(6) For $p \in P$, the orbits $\mathcal{O}_{p}=\left\{F(p) \mid F \in I_{(q, \omega)}(P)\right\}$ are closed submanifolds of $P$, and the maps

$$
\frac{I_{(g . \omega)}(P)}{\left(I_{(g . \omega)}(P)\right)_{p}} \rightarrow \mathcal{O}_{p} \subseteq P, \quad[F] \mapsto F(p)
$$

are diffeomorphisms onto the orbits.

(7) If $P(M, G)$ is compact, then $I_{(g,(\omega)}(P)$ is a compact Lie transformation group. 
Proof. (1) and (3). As in Sect. 6 and Theorem 6.2, let $\gamma$ be a positive-definite inner product on $6 \mathfrak{5}$. Then $\hat{g}=\pi^{*} g+\gamma \cdot(\omega \otimes \omega)$ is a Riemannian metric on $P$. If $F \in I_{(g, \omega)}(P)$,

$$
F^{*} \hat{g}=\pi^{*}\left(f^{*} g\right)+\gamma \cdot\left(F^{*} \omega \otimes F^{*} \omega\right)=\hat{g},
$$

so $I_{(g, \omega)}(P)$ is a closed subgroup of $I_{\hat{g}}(P)$. Thus $I_{(g, \omega)}(P)$ is a Lie transformation group on $P$, and the action is proper.

(2): If $F_{\lambda}$ is a one-parameter subgroup of $I_{(g, \omega)}(P)$ with generator $Z \in \mathfrak{X}_{G}(P)$, then $Z$ is a complete vector field and $F_{\lambda}^{*} \omega=\omega$ and $f_{\lambda}^{*} g=g$, where $f_{\lambda}=\hat{\pi}\left(F_{\lambda}\right)$. Thus $L_{Z} \omega=0$ and $L_{X} g=0$.

Conversely, if $Z \in \mathscr{I}_{(g, \omega)}(P)$, then by definition of $\mathscr{I}_{(g, \omega)}(P), Z$ is complete and $L_{Z} \omega=0$ and $L_{X} g=0$. Let $F_{\lambda}$ be the flow of $Z$ and $f_{\lambda}=\hat{\pi}\left(F_{\lambda}\right)$ the flow of $X=\pi_{*} Z$. Then $F_{\lambda}^{*} \omega=\omega$ and $f_{\lambda}^{*} g=g$. Thus $F_{\lambda} \in I_{(g, \omega)}(P)$.

(4): Note that since $\operatorname{dim} P=n+k$, we have the immediate rough estimate

$$
\operatorname{dim} I_{(g, \omega)}(P) \leqq \frac{1}{2}(n+k)(n+k+1) .
$$

This can be refined as follows. For $Z \in \mathscr{I}_{(g, \omega)}(P)$, let $X=\pi_{*} Z \in \mathscr{I}_{g}(M)$, the space of complete Killing vector fields on $M$. Let $O(M)$ denote the orthonormal frame bundle of $M$, and for $p \in P$ and $u \in O(M)$, consider the map

$$
\mathscr{I}_{(g, \omega)}(P) \rightarrow\left(\mathfrak{5} \times T_{u}(O(M)), \quad Z \mapsto(\omega(p) \cdot Z(p), \hat{X}(u)),\right.
$$

where $\hat{X}(u)$ denotes the natural lift of $X$ to $O(M)$. This map is injective, for if $\omega(p) \cdot Z(p)=0$ and $\hat{X}(u)=0$, then $X(x)=0$ and $T_{x} X=0$, where $x$ is the base point of $u$. Then since $X$ is a Killing vector field, $X=0$. Thus $Z$ is a vertical vector field, and so $Z \in \mathscr{I}_{\omega}(P)$. Since $\omega(p) \cdot Z(p)=0, Z=0$. Since the above map is injective,

$$
\operatorname{dim} \mathscr{I}_{(g, \omega)}(P) \leqq \operatorname{dim} G+\operatorname{dim} T_{u}(O(M))=k+\frac{1}{2} n(n+1) .
$$

(5): Since the action is proper, the isotropy subgroups are compact. Alternately, $\left(I_{(g, \omega)}(P)\right)_{p_{0}}$ is a closed subgroup of the compact isotropy group $\left(I_{\hat{g}}(P)\right)_{p_{0}}$ $=\left\{F \in I_{\hat{g}}(P) \mid F\left(p_{0}\right)=p_{0}\right\}$.

(6): This is a consequence of the properness of the action (Abraham-Marsden [1978]).

(7): If $P(M, G)$ is compact, $I_{\hat{g}}(P)$ is a compact transformation group. Since $I_{(g, \omega)}(P)$ is a closed subgroup, it is also compact.

Remarks. 1. Note that in contrast to $\mathscr{I}_{\omega}(P)$, where infinitesimal vertical symmetries of $\omega$ are automatically complete, infinitesimal symmetries $Z$ of $\mathscr{I}_{(g, \omega)}$ (i.e., $L_{Z} \omega=0$ and $\left.L_{X} g=0\right)$ are not necessarily complete, so the Lie algebra $\mathscr{I}_{(g, \omega)}(P)$ must be restricted to the space of complete infinitesimal symmetries.

2. Another difference between the groups $I_{\omega}(P)$ and $I_{(q, \omega)}(P)$ is that the action of $I_{(g, \omega)}(P)$ on $P$ is not necessarily free. This is because if $F \in I_{(g . \omega)}(P)$ with $F\left(p_{0}\right)=p_{0}$, then $f\left(x_{0}\right)=x_{0}$, where $f=\hat{\pi}(F) \in I_{g}(M)$ and $x_{0}=\pi\left(p_{0}\right)$. But an isometry which fixes a point need not be the identity. Thus $F$ need not cover the identity, and thus Proposition 2.1 cannot be applied. Thus $F$ fixing a point $p_{0} \in P$ is not sufficient to cause $F$ to be the identity (see Example 7.2 below).

3. Since the action of $I_{(g, \omega)}(P)$ is not necessarily free, the orbit space $\frac{P}{I_{(g, \omega)}(P)}$ is 
not necessarily a manifold. However, since the action is proper, it is a Hausdorff space.

Here's an example of the non-freeness of the action of $I_{(g, \omega)}(P)$ on $P$.

7.2. Example. Let $S^{n}$ be the standard unit sphere in $\mathbb{R}^{n+1}$ with standard metric $g_{0}, x_{0}$ the north pole, and $f \in I_{g_{0}}\left(S^{n}\right)$, an isometry fixing $x_{0}$ with $f \neq \mathrm{id}_{M}$. Let $P\left(S^{n}, G\right)$ $=S^{n} \times G$ be the product bundle, and let $\omega_{0}=\pi_{2}^{*} \theta$ the canonical flat connection on $P$ (see Example 3.2). Let

$$
F: S^{n} \times G \rightarrow S^{n} \times G, \quad(x, a) \mapsto(f(x), a) .
$$

Then $F \in \operatorname{AUT}(P), F$ covers $f$, and

$$
F^{*} \omega_{0}=F^{*} \pi_{2}^{*} \theta=\left(\pi_{2} \circ F\right)^{*} \theta=\pi_{2}^{*} \theta=\omega_{0},
$$

so that $F \in I_{\left(g_{0}, \omega_{0}\right)}(P)$. Also $F$ fixes $\left(x_{0}, a\right), F\left(x_{0}, a\right)=\left(f\left(x_{0}\right), a\right)=\left(x_{0}, a\right)$, but $F \neq$ id on $S^{n} \times G$.

For $Z \in \mathfrak{X}_{G}(P)$ and $\omega \in \mathscr{C}(P)$, the Lie derivative $L_{Z} \omega \in A^{1}(P,(\mathfrak{G})$ can be further examined. Let $Z=T+\bar{X}$ be the decomposition of $Z$ into its vertical and horizontal part. Here $T \in \mathfrak{X}_{G}^{\text {vert }}(P)$ is defined by $T(p)=T_{e} \Phi_{p} \cdot(\omega(p) \cdot Z(p))$, and $\bar{X}$ is the horizontal lift of $X=\pi_{*} Z \in \mathfrak{X}(M)$.

7.3. Proposition. Let $Z \in \mathfrak{X}_{G}(P), \omega \in \mathscr{C}(P)$, and let $Z=T+\bar{X}$ the decomposition of $Z$ into $G$-invariant vertical and horizontal vector fields. Let $\varphi_{T}=\omega(T) \in C_{\mathbf{A d}}^{\infty}(P,(\mathfrak{5})$ correspond to $T$ according to Proposition 1.1, and let $\Omega=D \omega \in A^{2}(P,(5)$ be the curvature of $\omega$. Then $L_{Z} \omega=D \varphi_{T}+i_{\bar{X}} \Omega$.

Proof. By Lemma 1.4, $L_{T} \omega=D \varphi_{T}$. Since $\omega(\bar{X})=0$,

and also

$$
L_{X} \omega=i_{\bar{X}} d \omega+d i_{\bar{X}} \omega=i_{\bar{X}} d \omega,
$$

Thus

$$
i_{\bar{X}} \Omega=i_{\bar{X}} d \omega+\frac{1}{2} i_{\bar{X}}([\omega \wedge \omega])=i_{\bar{X}} d \omega
$$

$$
L_{Z} \omega=L_{T+\bar{X}} \omega=L_{T} \omega+L_{\bar{X}} \omega=D \varphi_{T}+i_{\bar{X}} d \omega=D \varphi_{T}+i_{\bar{X}} \Omega .
$$

Thus the Lie algebra $\mathscr{I}_{(g, \omega)}(P)$ can be described by

$$
\mathscr{I}_{(g . \omega)}(P)=\left\{Z \in \mathfrak{X}_{G}(P) \mid Z \text { is complete and } D \varphi_{T}+i_{\tilde{X}} \Omega=0 \text { and } L_{X} g=0\right\} \text {, }
$$

where $\varphi_{T}=\omega(T)=\omega(Z)$, and $X=\pi_{*} Z$. This description is important in Yang-Mills field theories (see e.g. Forgacs-Manton [1980]; Jackiw-Manton [1979]; Fischer [1985]).

Finally we remark that in Lagrangian gauge field theories, the Lie group $I_{(g, \omega)}(P)$ plays an important role as the generator of global conservation laws for matter fields coupled to the fixed parameter fields $(g, \omega)$. This is analogous to the role played by $I_{\omega}(P)$ as the generator of global internal conservation laws, and to the role played by $I_{g}(M)$ as the generator of the global spacetime conservation laws. Here $g$ is usually a Lorentz signature metric (see Fischer $[1984,1985]$ for more details). 


\section{References}

1. Abraham, R., Marsden, J.: Foundation of mechanics, second edition. Reading, MA: Benjamin/Cummings 1978

2. Atiyah, M.F., Hitchin, N.J., Singer, I.M.: Self-duality in four-dimensional Riemannian geometry. Proc. R. Soc. Lond. A 362, 425-461 (1978)

3. Atiyah, M.F., Jones, J.D.S.: Topological aspects of Yang-Mills theory. Commun. Math. Phys. 61, 97-118 (1978)

4. Bleecker, D.: Gauge Theory and Variational Principles. Reading, MA: Addison-Wesley 1981

5. Daniel, M., Viallet, C.M.: The geometrical setting of gauge theories of the Yang-Mills type. Rev. Mod. Phys. 52, 175-197 (1980)

6. Fischer, A.: A unified approach to conservation laws in general relativity, gauge theories, and elementary particle physics. Gen. Relativ. Gravitation 14, 683-689 (1982)

7. Fischer, A.: Conservation laws in gauge field theories. In: Differential topology, global analysis on manifolds, and their applications. Rassias, G.M., Rassias, T. (eds.). Berlin, Heidelberg, New York: Springer 1985

8. Fischer, A.: A geometric approach to gauge field theories. Lecture Notes in Physics. Berlin, Heidelberg, New York: Springer (to appear)

9. Forgacs, P., Manton, N.S.: Space-time symmetries in gauge theories. Commun. Math. Phys. 72, 15-35 (1980)

10. Greenberg, M., Harper, J.: Algebraic topology, a first course. Reading, MA: Benjamin/Cummings 1981

11. Helgason, S.: Differential geometry and symmetric spaces. New York: Academic Press 1962

12. Jackiw, R., Manton, N.S.: Symmetries and conservation laws in gauge theories. Nucl. Phys. B 158, 141 (1979)

13. Kobayashi, S.: Transformation groups in differential geometry. Berlin. Heidelberg, New York: Springer 1972

14. Kobayashi, S., Nomizu, K.: Foundations of differential geometry. Vol. I. New York: Intersceince 1963

15. Kobayashi, S., Nomizu, K.: Foundations of differential geometry. Vol. II. New York: Interscience 1969

16. Mitter, P.K.: Geometry of the space of gauge orbits and the Yang-Mills dynamical system. In: Recent developments in gauge theories (Cargese Lectures, 1979). Hooft, G. et al. (eds.). New York: Plenum Press 1980

17. Myers, S.B., Steenrod, N.: The group of isometries of a Riemannian manifold. Ann. Math. 40, 400-416 (1939)

18. Poor, W.A.: Differential Geometric Structures. New York: McGraw-Hill 1981

19. Rawnsley, J.H.: Differential geometry of instantons. Communications of the Dublin Institute for Advanced Studies, Series A (Theoretical Physics), No. 25, 1978

20. Singer, I.M.: Some remarks on the Gribov ambiguity. Commun. Math. Phys. 60, 7-12 (1978)

21. Spanier, F.: Algebraic topology. New York: McGraw-Hill 1966

22. Trautman, A.: On groups of gauge transformations. In: Geometrical and topological methods in gauge theories. Harnad, J.P., Shnider, S. (eds.). Lecture Notes in Physics, Vol. 129. Berlin, Heidelberg, New York: Springer 1980

23. Warner, F.W.: Foundations of differentiable manifolds and Lie groups. Glenview, Illinois: Scott, Foresman 1971

Communicated by S. W. Hawking

Received June 17, 1985; in revised form April 23, 1987 